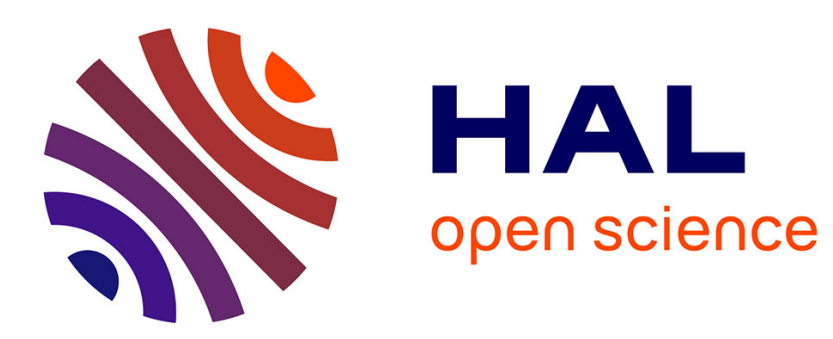

\title{
Vers une sémiotique du sensible: des couleurs en discours et en pratiques
}

Danièle S. Dubois, Caroline Cance

\section{To cite this version:}

Danièle S. Dubois, Caroline Cance. Vers une sémiotique du sensible: des couleurs en discours et en pratiques. Histoire Epistémologie Langage, 2012, 34 (1), pp.63-95. 10.3406/hel.2012.3237 . hal02342947

\section{HAL Id: hal-02342947 \\ https://hal.science/hal-02342947}

Submitted on 19 Feb 2021

HAL is a multi-disciplinary open access archive for the deposit and dissemination of scientific research documents, whether they are published or not. The documents may come from teaching and research institutions in France or abroad, or from public or private research centers.
L'archive ouverte pluridisciplinaire HAL, est destinée au dépôt et à la diffusion de documents scientifiques de niveau recherche, publiés ou non, émanant des établissements d'enseignement et de recherche français ou étrangers, des laboratoires publics ou privés. 


\section{Vers une sémiotique du sensible : des couleurs en discours et en} pratiques

Danièle Dubois, Caroline Cance

\section{Citer ce document / Cite this document :}

Dubois Danièle, Cance Caroline. Vers une sémiotique du sensible : des couleurs en discours et en pratiques. In: Histoire Épistémologie Langage, tome 34, fascicule 1, 2012. La linguistique cognitive : histoire et épistémologie. pp. 63-95; doi : https://doi.org/10.3406/hel.2012.3237

https://www.persee.fr/doc/hel_0750-8069_2012_num_34_1_3237

Fichier pdf généré le 16/01/2019 


\begin{abstract}
We examine the relations between cognitive psychology and linguistics, restrictively tracked through the precise analysis of colour terms and colour categories, as an emblematic issue in cognitive semantics. We first proceed to a precise exploration of the lexical resources involved in referring to colours (in French), within the diversity of technical devices representing colours, and then explore the diversity of syntactic inscriptions of colour terms in French in descriptive discourses. The diversity of linguistic devices involved in colour naming and their syntactic inscriptions in discourse allow us identifying a diversity of colour conceptualisations. Connecting a semantic theory of languages and a psychological theory of categorisation challenges the mainstream of cognitive linguistics by proposing a semiotic theory of cultural cognition.
\end{abstract}

\title{
Résumé
}

Nous examinons comment la linguistique cognitive aborde les relations entre langage et pensée à partir de l'exemple emblématique des couleurs. L'analyse linguistique des formes lexicales référant aux couleurs disponibles en langue et repérées dans différents types de corpus (dénominations de pastilles colorées, discours descriptifs de la qualité visuelle), couplée avec une théorie psychologique de la catégorisation, conduit à identifier une diversité de conceptualisations et de modes de référenciation de la couleur. Ceci nous amène à discuter la position naturalisante de la sémantique cognitive et à proposer une théorie linguistique cognitive "située » où s'articulent une sémantique des langues et de la construction du sens en discours, et une théorie psychologique des catégories du sensible dans une sémiotique cognitive des cultures. 


\title{
VERS UNE SÉMIOTIQUE DU SENSIBLE : \\ DES COULEURS EN DISCOURS ET EN PRATIQUES
}

\author{
Danièle Dubois* \\ Caroline Cance** \\ *LCPE / LAM \\ **LLL - Université d'Orléans
}

\begin{abstract}
Résumé
Nous examinons comment la linguistique cognitive aborde les relations entre langage et pensée à partir de l'exemple emblématique des couleurs. L'analyse linguistique des formes lexicales référant aux couleurs disponibles en langue et repérées dans différents types de corpus (dénominations de pastilles colorées, discours descriptifs de la qualité visuelle), couplée avec une théorie psychologique de la catégorisation, conduit à identifier une diversité de conceptualisations et de modes de référenciation de la couleur. Ceci nous amène à discuter la position naturalisante de la sémantique cognitive et à proposer une théorie linguistique cognitive « située » où s'articulent une sémantique des langues et de la construction du sens en discours, et une théorie psychologique des catégories du sensible dans une sémiotique cognitive des cultures.
\end{abstract}

\section{Mots clefs}

Sémantique cognitive, cognition située, couleurs, catégories, lexique, discours

\section{Abstract}

We examine the relations between cognitive psychology and linguistics, restrictively tracked through the precise analysis of colour terms and colour categories, as an emblematic issue in cognitive semantics. We first proceed to a precise exploration of the lexical resources involved in referring to colours (in French), within the diversity of technical devices representing colours, and then explore the diversity of syntactic inscriptions of colour terms in French in descriptive discourses. The diversity of linguistic devices involved in colour naming and their syntactic inscriptions in discourse allow us identifying a diversity of colour conceptualisations. Connecting a semantic theory of languages and a psychological theory of categorisation challenges the mainstream of cognitive linguistics by proposing a semiotic theory of cultural cognition.

\section{Keywords}

Cognitive semantics, situated cognition, colours, categories, lexicon, discourse

\section{INTRODUCTION}

Cet article a pour objectif principal d'examiner les relations entre langage et pensée au sein des sciences cognitives en partant de l'articulation entre les champs de la linguistique et de la psychologie. Il ne s'agit pas de traiter ici ni de la constitution de la psycholinguistique comme champ autonome au sein de la psychologie expérimentale, ni de la représentation des connaissances et de la mémoire sémantique en Intelligence Artificielle qui s'avéraient être les questions centrales des années 1980 et 1990 (Dubois 1989), mais de préciser et analyser l'évolution 
actuelle du positionnement des recherches psychologiques et linguistiques relatives à la sémantique lexicale qui configurent les relations entre langue et cognition. ${ }^{1}$

Dans un premier temps nous procèderons à un examen critique de cette question dans l'évolution générale des recherches en linguistique cognitive, à travers le programme de «naturalisation » des phénomènes cognitifs et plus récemment la problématique de l'«embodied cognition ». Cette analyse s'appuiera sur les travaux en sémantique lexicale, à partir de l'analyse des recherches emblématiques relatives aux catégories (cognitives et lexicales) des couleurs. Nous verrons comment depuis les années 1980 en effet, la cognition se «naturalise », en réaction à une (la ?) conception « intellectuelle » logico-formelle issue de la métaphore computationnelle (Fodor 1983). Nous reprendrons des éléments d'analyse du concept de naturalité dont nous avons montré (Dubois \& Resche-Rigon 1995) les ambiguïtés ou tout au moins la polysémie, au sein même d'une conception de la cognition comme « traitement de l'information ». Ces orientations laissent cependant « un gap », à savoir le rôle des langues et des pratiques langagières dans l'articulation entre l'individuel et le collectif, tant dans la construction collective des connaissances que dans l'appropriation individuelle du « culturel ». Nous proposerons, à travers une analyse du domaine des couleurs, de préciser ces lieux d'articulation, dans le champ de la psychologie, dans le cadre d'une sémiotique des catégories cognitives des couleurs et, dans le champ linguistique, d'une sémantique lexicale de la dénomination des couleurs relevant cette fois d'une linguistique des langues. Faute de prendre en compte le fait que chaque discipline appréhende ses objets et construit leurs propriétés d'une façon spécifique, les débats actuels en sciences cognitives restent improductifs. Ils demeurent le plus souvent ancrés dans une cognition par trop générique, où manquent des chaînons qui fondent une cognition située dans les pratiques humaines et les ressources des langues, et qui amènent à reconsidérer du même coup l'opposition entre un individuel biologique (donc ?) universel confronté à la réalité physique et un « culturel » indifférencié, sinon globalisé. ${ }^{2}$

Nous développerons cette argumentation à travers une reprise des travaux linguistiques sur les mots de couleurs, situés cette fois dans la diversité de pratiques ordinaires de la couleur, incluant ainsi les discours où s'inscrivent les mots de couleurs. Cela nous amènera à prendre également en compte l'inscription matérielle des couleurs dans les dispositifs de présentation de la couleur (tant dans les situations expérimentales que dans les situations quotidiennes) et d'identifier comment ces artefacts matériels sont investis et suscitent différents processus de sémiotisation qui construisent les couleurs comme connaissances (et non plus comme catégories «naturelles »), ce au travers de deux types de situations :

- dans une situation de dénomination analogue au protocole de Berlin et Kay, « désuniversalisé » car ancrée dans des pratiques expertes et quotidiennes (utilisation de nuanciers de couleurs pour différentes finalités commerciales);

1 Le déplacement du questionnement de langage à langue et de pensée à cognition est délibérément pris comme symptôme de l'évolution de la thématique que nous présentons ici.

2 «Identifying semantic category as the verbal response to physical stimuli, naturalism would confine the problem of meaning with the endemic antinomy of a world-less subject confronting a tought-less object: the antique dualism of mind and matter. »(Sahlins 1976). 
- dans des pratiques de discours « ordinaires », au sein d'une étude sur l'évaluation de la qualité visuelle d'espaces complexes, réels ou virtuels.

Ces travaux nous permettront de mettre en évidence, à travers l'exemple des couleurs, les processus de sémiotisation relevant de co-constructions du sens dans les discours et les pratiques, et qui affectent différentes fonctions référentielles à une «même » forme lexicale dans le cadre d'une sémantique des langues et d'une théorie psychologique des catégories du sensible.

\section{LINGUISTIQUE COGNITIVE : LANGAGE ET COGNITION}

Un premier bilan proposé dans le numéro d'HEL de 1989 (Rastier 1989), suivi de plusieurs analyses et revues a déjà tracé l'évolution récente de la linguistique cognitive (voir entre autres Rastier 1991, 1993, 2005, Geeraerts 1993, AchardBayle \& Paveau 2007, Nyckees 2007, pour ne rester qu'au niveau francophone), principalement d'un point de vue critique en regard de l'histoire des théories sémantiques, mais tenant généralement pour acquis les données de la psychologie cognitive. Nous nous proposons dans un premier temps de resituer l'articulation entre psychologie et linguistique dans le cadre plus général des recherches en linguistique cognitive des dernières décennies. Notre propre lecture de ces recherches nous amène à reprendre nombre des éléments de discussions déjà pris en compte en nous attachant toutefois à spécifier les articulations de la sémantique linguistique aux cadres théoriques et méthodologiques et donc aux « données » de la psychologie sur lesquelles s'appuie la recherche linguistique en sémantique cognitive.

\subsection{Le cognitif : forme logique ou contenu expérientiel?}

Ainsi, nous repartirons dans un premier temps du constat, maintes fois décrit (cf. Cadiot \& Visetti 2001, Rastier 2005, Achard-Bayle \& Paveau 2007 parmi d'autres), de l'évolution divergente des recherches linguistiques qui s'est manifestée dès les années 1970 en opposition au « cognitivisme orthodoxe » chomskyen, développé au MIT. La « sémantique générative ${ }^{3}$ » d'abord (cf. Dubois-Charlier \& Galmiche 1972) puis la linguistique cognitive développées sur la côte californienne vont faire évoluer en particulier la sémantique lexicale : «c'est en effet la linguistique cognitive qui a renouvelé les recherches en sémantique lexicale linguistique en introduisant le modèle prototypique de la catégorisation » (Geerearts 1993, p. 112) (sur le rapport à la sémantique générative voir également Fortis (2011) et ce numéro).

En effet, ainsi que nous l'analysions dans la revue de 1989, la divergence au sein de l'espace pluridisciplinaire des sciences cognitives naissantes au cours des années 1970 et 1980, s'est manifestée dans une opposition entre une conception formelle et logiciste du langage et du fonctionnement psychologique développée sur la Côte Est des États-Unis et une posture plus «naturaliste » des phénomènes linguistiques et psychologiques. Il s'agissait pour les premiers, de

3 qui préfigure la linguistique cognitive. 
tester expérimentalement la « réalité psychologique » des règles linguistiques qui régissent la grammaire universelle. Pour Chomsky en effet, l'objectif de la linguistique est explicitement « de développer l'étude de la structure linguistique comme un chapitre de la psychologie humaine. [...] la première tâche du psychologue (étant) de découvrir le schéma inné qui caractérise la classe des langues potentielles qui définit l'essence du langage humain. Cette tâche est du ressort de la branche de la psychologie humaine connue sous le nom de linguistique » (Chomsky 1968/1970 - trad. fr., p. 101 et 128). De leur côté, les linguistes de la côte Ouest ont été amenés à développer « une orientation carrément psychologique et cognitive de l'étude sémantique »(Geeraerts 1993, p. 117). Cette position s'est clairement affirmée dans les recherches dans le domaine cognitif de l'espace (pour une synthèse voir Fortis 1996) et sur la métaphore (Lakoff \& Johnson 1980). Ainsi les espaces mentaux (Fauconnier 1985, Lakoff 1987) sont définis comme une mentalisation d'un espace objectif intériorisé. Le sens lexical demeure cependant référentiel, mais avec un déplacement d'une référence au «monde tel qu'il est », tel qu'il est objectivé par les sciences de la nature, à une référence au «monde tel qu'il est représenté » à travers l'expérience humaine.

Ces deux orientations en linguistique cognitive s'accordent donc à revendiquer toutes deux un certain psychologisme en référant aux fondements « mentaux » des phénomènes linguistiques. Nous voudrions cependant examiner ici de quelle conception du mental ou du cognitif il s'agit, et ce à partir de différentes équivalences et couples d'opposition qui traversent différemment les sciences cognitives et dessinent différentes conceptions du « mental ».

\subsection{De la réalité psychologique aux catégories naturelles}

En déplaçant la recherche linguistique des règles syntaxiques de la grammaire universelle aux phénomènes sémantiques et lexicaux et en essayant «d'appliquer systématiquement la méthodologie de la grammaire générative à un ensemble toujours plus grand de données » (Lakoff 1971, note 1), la sémantique générative, prélude à la linguistique cognitive de la côte ouest, va «naturaliser » la grammaire, en reliant les formes linguistiques des langues « naturelles $»^{4}$ (natural languages) d'abord à des formes logiques elles aussi «naturelles », « qui ne feraient pas d'affirmation sur l'univers, mais (qui) feraient des affirmations sur la manière dont les être humains conçoivent l'univers » (Lakoff 1970, cité par DuboisCharlier \& Galmiche 1972, p. 77). Ceci aura également des conséquences que nous n'aborderons pas ici quant à l'articulation entre lexique, sémantique et syntaxe et la remise en cause du postulat chomskyen concernant l'autonomie de la syntaxe (voir parmi de nombreuses autres les analyses de Talmy (1992) et de Langacker (1987)).

Cette évolution en linguistique dans la recherche d'universaux caractéristiques tant des propriétés des langues telles que la polysémie ou la métaphore que de celles de l'esprit humain va alors se développer (en particulier à Berkeley) en

4 Langues naturelles s'opposant ici à langage formel : on pourra noter qu'il s'agit peut-être d'un calque sur l'anglais qui nécessite de lever une ambiguité due à la polysémie de language. On remarquera d'ailleurs la coexistence en français de TAL - Traitement Automatique des Langues et de TALN - Traitement Automatique du Langage Naturel comme équivalent à NLP (Natural Language Processing). 
étroite interaction avec l'évolution de la psychologie cognitive. La recherche psychologique va déplacer l'analyse d'une cognition considérée par trop « intellectuelle » à une approche qualifiée d' « écologique » (Neisser 1987) en particulier, à travers le modèle prototypique de la catégorisation et des structures cognitives des catégories elles aussi « naturelles ».

Cependant, le concept de naturalité, dans son opposition première au caractère formel va se colorer d'une diversité de sens, en relation précisément avec les fondements (ou présupposés) mentalistes de la sémantique. On renverra à Dubois et Resche-Rigon (1995) pour une analyse de cette polysémie du qualificatif de « naturel » que nous avons déjà mentionnée et que nous ne reprendrons que partiellement ici pour la préciser dans l'articulation de la linguistique et de la psychologie en sémantique cognitive.

1.2.1 Naturel expérientiel et perceptif

En récusant l'approche formelle, et en revendiquant la naturalité des langues et des représentations cognitives fondées dans l'expérience humaine, la sémantique va s'appuyer sur la connaissance des processus perceptifs. Selon Rosch qui a introduit les recherches sur les catégories naturelles en psychologie (Rosch, 1973), c'est à partir des processus perceptifs qui permettent le repérage des discontinuités dans la distribution des propriétés de l'environnement que vont se construire les catégories naturelles auxquelles ensuite il sera possible de donner des noms :

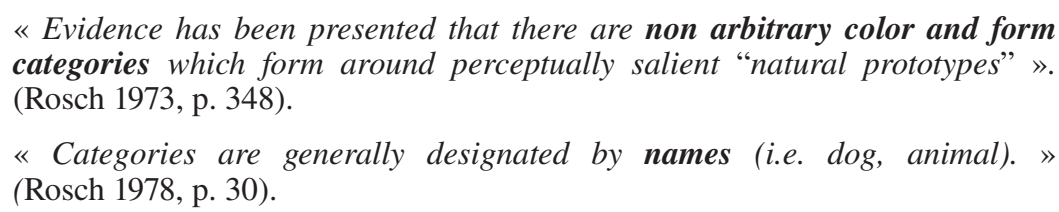

Les catégories cognitives se trouvent ainsi déterminées par le traitement perceptif qui est conçu comme extraction d'une information objectivement présente dans le monde, selon une conception ascendante de la perception sur laquelle nous reviendrons plus loin, en accord avec la reprise en psychologie du modèle classique du cognitivisme contemporain qui considère l'humain comme un « système de traitement de l'information » (Lindsay \& Norman 1972).

1.2.2 Naturel expérientiel et cognition « embodied»

Ceci pourrait sembler contradictoire avec le souci de renouvellement d'une conception de la cognition jugée par trop formelle, car trop influencée par la métaphore computationnelle. Et ce d'autant que cette rupture avec le cognitivisme classique est revendiquée en inscrivant le naturel, l'expérientiel, dans une cognition « embodied» (incarnée ? incorporée ?5) et non plus indépendante de son inscription matérielle (voir l'approche développée initialement par Varela et al., 1991 puis reprise entre autres par Lakoff \& Johnson 1999). Alors que le cognitivisme classique

5 Devant les difficultés rencontrées pour traduire embodied, les traductions disponibles (incorporée, incarnée) nous semblant partielles et non satisfaisantes car renvoyant aux concepts de chair et de corps en français qui nous paraissent quelque peu différents du concept de body en anglais, nous faisons le choix de conserver le mot dans sa version originale. 
postule précisément que le traitement de l'information est indépendant du support matériel fut-il biologique, organique ou physico-chimique, l'expérientiel embodied va ainsi se trouver biologisé. En dernière instance, les formes linguistiques (le lexique en l'occurrence) se trouvent elles-mêmes (et à nouveau, comme dans la tradition chomskyenne) contraintes par des déterminismes biologiques à travers un raisonnement qui sauvegarde l'universalité dans une cognition peut-être incarnée, mais « dépsychologisée », puisque reflétant (incarnant) à la fois la structure et les propriétés du monde.

1.2.3 Prototype : catégorie « naturelle » ou connaissance acquise

Cette réduction des catégories naturelles à des déterminismes universels innés, biologiques est d'autant plus surprenante (voire encore une fois contradictoire) que l'on a pu montrer (Dubois \& Resche-Rigon 1995) que les catégories naturelles dans la théorie de E. Rosch relèvent davantage de connaissances élaborées au cours de l'histoire d'une culture (la nôtre - occidentale). Si elles peuvent être désignées de catégories « naturelles », c'est donc comme catégories des sciences naturelles, c'est-à-dire des catégories référant aux connaissances acquises au cours de l'histoire relatives aux objets de la nature, et non comme il le semblait aux propriétés naturelles (psychologiques) de l'activité classificatoire, cette tradition classificatoire étant d'ailleurs elle-même historique et culturelle (voir Tort 1989, Vignaux 1999, Selosse 2008 pour les aspects linguistiques de l'histoire des classifications).

Comme nous l'avons déjà discuté par ailleurs (Dubois \& Resche-Rigon 1993), le concept de prototype issu d'une théorie psychologique de la catégorisation (Rosch 1978) et repris en linguistique cognitive (Lakoff 1987, Langacker 1987 ; voir aussi Geeraerts (1993) déjà cité, parmi de nombreux autres), demeure particulièrement non explicite et ambigu quant à son statut psychologique et son articulation avec le concept de représentation (lui-même polysémique). On a pu ainsi montrer une grande imprécision dans l'utilisation du concept de prototype, bien souvent indifférencié de celui de stéréotype, c'est-à-dire de catégories élaborées à travers le développement des connaissances (sociales, collectives) dans la tradition classificatoire de la culture occidentale. Lorsque la distinction est faite conceptuellement, comme c'est le cas dans le champ linguistique (Lakoff 1987, p. 85-87), elle demeure non explicite d'un point de vue psychologique, sans différencier les processus d'apprentissage à travers une expérience sensorielle individuelle et les processus d'acquisition de connaissances (principalement scolaires) et de socialisation. Si la démonstration s'applique aux catégories « naturelles » en tant que catégories des sciences de la nature, elle peut devenir a fortiori valide concernant les catégories des artefacts (les meubles, les armes, etc.). Nous montrerons ci-après ( $\$ 2$.) que le statut de connaissances s'applique également aux catégories prétendument plus naturelles que sont les catégories perceptives des couleurs, comme d'ailleurs des autres catégories issues des autres modalités sensorielles (Dubois 2000 et 2007).

1.2.4 Naturel comme «basic »

L'introduction du naturel est également étroitement associé au concept de « catégories de base » (basic category) elles-mêmes identifiées à partir du repérage de termes 
de base (basic terms) en particulier dans la problématique de la catégorisation des couleurs (cf. Rosch d'abord avec son travail sur les Dani - Rosch-Heider 1971), mondialisée par les incontournables Berlin et Kay (1969). Naturel, comme basique renvoie alors à premier, aux constructions cognitives ou /et (indifféremment) aux formes lexicales («terms ») à partir desquelles vont se développer des catégories et dénominations plus élaborées, plus génériques et plus spécifiques (Berlin \& Kay 1969, Rosch et al. 1976). Naturel signifie alors également primitif : les termes de base sont les premiers présents dans toutes les cultures, même celles qualifiées de technologiquement « moins développées », avant toute « contamination » extérieure/ étrangère culturelle ${ }^{6}$. Associé à une hypothèse sur l'évolution de deux à onze termes de base pour rendre compte de la diversité lexicale des langues, naturel renvoie ainsi à une conception adamique de l'histoire des langues (cf. discutée par ailleurs en particulier Eco 1994, Resche-Rigon 2009).

Cette réduction du naturel aux aspects basique, perceptif et expérientiel, physiologique dont nous venons de rendre compte permet de garantir le caractère universel défendu par le cognitivisme orthodoxe. Si le concept de catégorie naturelle permet de « réincarner » une cognition auparavant par trop formelle et abstraite, celui-ci ne remet pas en cause la nature des catégories qui s'avèrent de fait non pas des représentations individuelles issues de l'expérience perceptive mais demeurent des connaissances construites collectivement et historiquement dans une culture qui sert de référence à l'évaluation des autres.

\subsection{Questions de méthodes}

Malgré l'intérêt renouvelé en linguistique cognitive pour l'étude des relations entre langue et pensée, la démarche reste cependant inscrite dans la tradition chomskyenne en particulier sur le plan des méthodes de questionnement, avec notamment le recours à des exemples construits ou provoqués en ce qui concerne les phénomènes syntaxiques, mais aussi sémantiques. En ce qui concerne le lexique et plus particulièrement les catégories lexicales des couleurs, comme des différentes modalités sensorielles, on assiste à la généralisation de procédures expérimentales visant à identifier pour chaque langue les termes de base («basic terms »), puis à les mettre en relation avec les catégories naturelles (« natural categories »), à partir d'un paradigme qui garantit la « scientificité » des données. C'est donc sur le terrain même de la constitution des données empiriques relevant des phénomènes psychologiques et langagiers qu'il convient également de porter la discussion de cette orientation prévalente en linguistique cognitive.

1.3.1 Une linguistique de l'exemple

Plusieurs auteurs (Rastier 2005, Achard Bayle \& Paveau 2007, Teubert 2010, parmi d'autres) constatent en effet que la majorité des travaux en linguistique cognitive demeurent basés sur quelques exemples de phrases (au mieux tirés d'un corpus, et le plus souvent issus de la créativité ou du répertoire personnel des auteurs). Ils ne prennent pas en compte les textes et discours attestés (c'est-à-dire ceux

6 Ce qui conduit à éliminer les termes d'emprunt dans le relevé des termes de base dans la diversité des langues (voir les critères de sélection des termes de bases dans le paradigme de Berlin et Kay analysé en section 2.1). 
effectivement produits dans diverses situations) et encore moins une typologie des genres de discours, restant en cela proche du projet des grammaires génératives décrit par Rastier comme étant « de générer l'ensemble des phrases grammaticales d'une langue (qui) est en lui-même irréaliste : une langue n'est pas faite de phrases, mais de textes, de genres et de discours » (Rastier 2005).

Ainsi comme le notent également Achard-Bayle et Paveau (2007, §5) :

« À un autre plan, épistémologique et méthodologique, il en résulte le plus souvent des travaux (en LC) qui perpétuent la tradition (structurale, générative plus ou moins consciemment revendiquée) d'une linguistique de la langue ${ }^{7}$ au détriment d'une linguistique des contextes ; une linguistique des énoncés (décontextualisés sinon fabriqués) au détriment d'une linguistique des textes et des discours. ${ }^{8} \gg$.

Ceci nous semble d'autant plus regrettable que les avancées de la linguistique de corpus qui travaille depuis les années 1970 sur les corpus attestés peuvent bénéficier à la linguistique cognitive et lui offrir des perspectives de renouvellement. Et ce en partant des formes linguistiques en tant que signes au sein de discours, au lieu de projeter un modèle sur des données et d'extraire quelques bonnes illustrations du modèle, comme le remarque Teubert, contrastant la linguistique de corpus et la linguistique cognitive (Teubert 2010, § 26) :

«A cognitive linguist would have developed a particular theory that is defined in categories and the relations obtaining between them, and any hypothesis they might want to test would be expressed in terms of these categories. [...] Basically researchers using this approach look in the corpus for examples for the structures or entities they have hypothesised. This is a use of corpus linguistics that has been termed by Elena Tognini Bonelli as 'corpus-based', as opposed to 'corpus-driven' for the bottom-up approach. » (Tognini Bonelli 2001).

Cette absence de prise en compte de la dimension corpus dans les problématiques cognitives est néanmoins à relativiser. Il semble en effet qu'un changement soit en train de s'opérer à en juger par l'apparition depuis quelques années de ces «nouvelles » thématiques au sein de conférences, journées d'étude, et publications. Ainsi en témoigne par exemple l'ouvrage de Glynn et Fischer (2010) ainsi que le tout récent appel à communication pour la journée d'étude 2012 de l'AFLICO (Association Française pour la Linguistique Cognitive) : multimodalité et corpus.

1.3.2 Une démarche expérimentale : un gage de scientificité ?

Parallèlement au caractère limité du travail sur corpus en linguistique cognitive, on observe une généralisation de la mise en place de procédures expérimentales qui prétendent garantir la scientificité des données pour combler la difficulté par exemple mentionnée par E. Sweetser (1990, p. 7 - cité par Achard-Bayle 2007, p. 52) en assurant à travers des stimuli contrôlés un référentiel «identique » mimétique des procédures des sciences expérimentales de la nature :

7 On peut se demander si cela ne relève alors pas davantage d'une sémantique du langage !

8 C'est nous qui soulignons. 
«Whether words for cultural categories are merely shaped by our understanding of culture, or whether they shape it as well, is a good deal harder to prove experimentally, since we can't (as done with colors) assume that the phenomena referred to are the same crosslinguistically ».

En effet, la question de savoir si ces phénomènes sont les mêmes (et pour qui ? cf. plus loin dans notre argumentation, l'analyse des pastilles de couleur du nuancier Munsell) ou non, reste entière. ${ }^{9}$ On peut s'étonner alors que Sweetser qui reconnaît la difficulté à mettre en place des protocoles expérimentaux permettant de vérifier la généricité ou au contraire la variation transculturelle et interlinguistique, mentionne néanmoins les couleurs comme contre-exemple, c'est à dire comme domaine dans lequel opère la magie de la procédure expérimentale pour régler définitivement le problème de l'universalité de la décomposition en catégories du spectre coloré. On verra cependant dans la partie 2 suivante qu'il n'en est rien.

Achard-Bayle note également que Wierzbicka est l'une des rares à remarquer dès 1988 la difficulté à mettre en place des procédures d'étude d'une «sémantique transculturelle » :
«It is a common place to say that every language embodies in its very structure a certain world-view, a certain philosophy [in the terms of] the Humboldtian (or Whorfian) thesis [...] To prove it in a rigorous and verifiable way, however, is quite a different matter. Scholars tend to treat the Humboldtian (or Whorfian) thesis [...] with suspicion and embarrassment. One suspects that this is precisely because while being obviously true it is at the same time notoriously difficult to prove.» (Wierzbicka 1988, p. 169).

Il apparaît ainsi d'autant plus fondamental de travailler précisément les questions de méthodologie. Nous nous attacherons dans la partie suivante (2.) à poser les limites du paradigme expérimental de Berlin \& Kay (1969). Utilisé en sémantique des couleurs comme exemple emblématique de procédure "contrôlée » sur le lexique, il a également été mis à contribution pour « prouver » expérimentalement l'universalité des catégories (cognitives) de couleurs en dépit de la diversité des langues et des cultures. En particulier nous montrerons, à travers l'analyse des différentes étapes de la mise en place de la procédure expérimentale, comment ce paradigme, qui vise à repérer les universaux linguistiques et cognitifs, opère en fait dans un domaine de validité restreint : le cadre de référence scientifique occidental. Et ce sont ces restrictions mêmes, en tant que catégories d'analyse a priori projetées sur l'expérience sensible qui produisent «l'universalité » des réponses.

Il s'agit donc maintenant de reprendre des arguments déjà développés en anthropologie (Howes 1991) et en linguistique (Lucy 1992, Foley 1997), tout comme en anthropologie des sciences (Latour 1993). Ceci nous permettra d'analyser le questionnement lui-même : la mise en place de procédures qui impliquent des stimuli, comme des objets non pas « naturels » mais comme artéfacts (cf. Straus dès 1935) directement et immédiatement investis dans leur perception même de significations culturelles, ainsi que les tâches demandées aux sujets, elles aussi codifiées culturellement. La discussion se trouve alors déplacée non seulement sur

9 à moins d'adhérer à un réalisme « basic » ou « naturel » ! 
le plan théorique mais également sur le plan empirique puisqu'il s'agit de discuter les preuves expérimentales de l'universalité des termes et des catégories basiques de couleur en rendant explicite l'ensemble des présupposés implicites sous-jacents à ce mode de questionnement.

\title{
TERMES ET CATÉGORIES DE BASE : DES COULEURS NATURELLES OU DES CONNAISSANCES?
}

Nous reprenons ici les développements exposés par ailleurs (Dubois \& Cance 2009), dans lesquels nous avons montré comment les procédures d'investigation révèlent, non seulement le caractère restreint d'une démarche empirique de sens commun, mais aussi les présupposés épistémologiques sur les concepts, les mots et les choses, qui la fondent ; en particulier parce que ces procédures limitent l'analyse aux « termes de base » indépendamment de leur contexte de questionnement ou d'énonciation et à leur adéquation à une certaine représentation des catégories de couleurs.

\subsection{Termes de base ou mots : veridical label ou partie du discours ?}

L'objectif de l'enquête linguistique (lexicale) de Berlin \& Kay (1969) était de montrer que derrière l'apparente diversité linguistique des manières de rendre compte des couleurs, on pouvait mettre en évidence des invariants attestant de l'unicité et donc de l'universalité de la segmentation du spectre coloré dans les mêmes catégories de base, quelles que soient les cultures. Les résultats obtenus les conduisaient à proposer une construction théorique basée sur deux hypothèses principales. Une première hypothèse s'attachait à vérifier l'existence universelle de " couleurs focales » que toutes les langues ayant des termes de couleurs posséderaient :

\begin{abstract}
« il apparaît actuellement que, bien que différentes langues encodent dans leurs vocabulaires des nombres différents de catégories de base pour les couleurs, un inventaire total et universel d'exactement onze catégories de base existe à partir desquels onze termes de base, ou moins, peuvent être extraits, pour toute langue. Les onze termes de base sont blanc - noir - rouge - jaune - vert - bleu - brun orange - gris - violet - rose. »(Berlin \& Kay 1969, p. 2, notre traduction).
\end{abstract}

La seconde renvoyait à une détermination génétique de l'ordre d'apparition des termes de base dans l'évolution des langues (cf. plus haut 1.2.4).

Selon Berlin \& Kay, un terme de base est défini comme :

« une forme qui présente minimalement les quatre caractéristiques suivantes : a) être monolexèmique : sa signification n'est pas prédictible de la signification de ses parties;

b) sa signification n'est pas incluse dans celle de tout autre terme de couleur ;

c) son application ne doit pas être réduite à une classe étroite d'objets ;

d) il doit être psychologiquement saillant pour les informateurs. Les indices de la saillance psychologique incluent, parmi d'autres, (1) la tendance à apparaître en début de liste suscitée de termes de couleur, (2) la stabilité de référence entre informateurs et entre occasions d'usage, et (3) l'occurrence dans les idiolectes de tous les informateurs » (ibid., p.6, notre traduction). 
Ainsi, en amont du test de leur hypothèse centrale, celle de la dépendance des formes linguistiques (les termes) vis-à-vis des structures cognitives, Berlin \& Kay éliminent au départ la diversité des modes d'inscription lexicale, en restreignant le lexique des couleurs aux seuls termes de base, et dans la définition même de ces termes. De plus, au sein même des critères linguistiques, ceux-ci relèvent à la fois des propriétés du système de la langue (critères morphologiques, tel a)), d'un certain usage de la langue, (tel c)), et de ses variations géographiques (tel d)) ou historiques (contact de langues et emprunts). Ces différents aspects correspondent chacun à des domaines théoriques et des conceptions de la langue et du langage dont la convergence au sein d'une analyse linguistique est postulée, mais qui relèvent davantage d'une conception de sens commun du lexique et du langage que d'une théorie linguistique experte explicite et dont la compatibilité et la pertinence, vis-à-vis du fonctionnement psychologique ne sont pas davantage explicitées (cf. Dubois 2009 ainsi que Guignard 2011).

\subsection{Les pastilles de couleurs : «natural colours » or dispositif technique?}

Le questionnement de Berlin et Kay s'appuie sur la référence à une représentation exacte des couleurs donnée par la « Munsell Chart». L'intérêt du nuancier Munsell comme référentiel (relativement) universel utilisé récemment comme standard commun aux peintres, industriels, cartographes voire pédologues (Latour 1993) n'est pas ici remis en cause..$^{10}$ Mais c'est son statut de référentiel unique pour évaluer la sémantique lexicale des termes de couleur qu'il conviendra de resituer en comparaison avec d'autres objets techniques (relativement) identiques (ou semblables) que sont d'autres nuanciers (cf. section 3). Latour insistait déjà sur le fait que la matérialité de la pastille colorée du nuancier Munsell qui sert d'intermédiaire pour qualifier comme identique une couleur portée par un autre objet de référence (une motte de terre en l'occurrence), fait que cette pastille appartient à la fois au monde des choses et à celui des signes.

De fait, si on résume l'analyse que nous avons pu faire (Dubois 2000, 2009), systématisant les remarques déjà nombreuses sur ce sujet (Saunders 1992, Lucy 1997, Levinson 2001, Wierzbicka 2008, par exemple), un tel tableau de pastilles colorées est un artéfact et donc le résultat d'une construction, qui découle :

- de processus complexes d'abstraction par notre culture de la notion de couleur comme dimension homogène, et indépendante des objets porteurs, en contradiction avec l'idée de fondements perceptifs « directs » de la réalité mondaine des couleurs ;

- de la constitution de la couleur comme une dimension continue, et de celle corrélative des couleurs comme catégories qui segmentent cet espace continu, sans faire référence à cette conception comme connaissance scientifique du monde physique (tardive : XVIII ${ }^{e}$ siècle), et non comme théorie psychologique de la perception ;

10 qui permet en particulier une standardisation qui faisait défaut aux nuanciers du XIX ${ }^{\mathrm{e}}$ qui utilisaient des brins de laine ou des papiers. 
- de son élaboration systématique dans une théorie physique de la lumière (mesurable donc en termes de longueur d'ondes par exemple et qui donne un sens à la disposition des pastilles, du rouge - les plus courtes longueurs d'ondes perçues, au violet - les plus longues) ;

- d'une discrétisation (finesse de catégorisation) en « catégories » (unités, segments...) et d'une systématisation dans leur ordonnancement, dont la motivation correspond autant aux contraintes de taille du dispositif (dans son ensemble et de chacune des pastilles) dans un intérêt pratique qu'à des hypothèses de discrimination neurophysiologique ou cognitive ;

- d'une reproduction dans des technologies qui permettent des paramétrages en accord avec la théorie scientifique, dont on peut noter qu'elle diffère par ailleurs des systématiques des couleurs proposées pour la pratique de la peinture, comme les rosaces des couleurs primaires (jaune, rouge, bleu et de leurs mélanges) ;

- enfin, d'une habituation sociale à choisir sur un nuancier une couleur, acquise dans notre culture pour de multiples usages (vestimentaires, décoratifs, voire scientifiques, etc.) que nous verrons plus en détail par ailleurs dans la partie 3 .

Ce référentiel couleur est ainsi de manière caricaturale la synthèse du savoir occidental sur la question. Il s'agit bien d'une construction élaborée par « une culture complexe » à travers « une technologie complexe $» .{ }^{11}$ La pastille de couleur comme représentation adéquate d'une couleur doit alors être conçue non comme une représentation « naturelle » de la couleur (ou des couleurs) mais comme un produit d'activités cognitives non seulement psychologiques, individuelles, mais aussi collectives dans l'élaboration d'une théorie scientifique de la couleur, et comme la (re)matérialisation d'une abstraction historiquement produite par une culture et un certain développement technologique (Indergand 1994). Ceci en lieu d'investir directement la couleur d'un statut ontologique soit comme une réalité du monde, soit comme une catégorie immuable ou première de l'entendement ou d'une vérité attestée parce que fondée dans la science physique. L'absence d'interrogation sur le statut de ce dispositif expérimental, de sa pertinence relativement à la notion même de couleur pour les individus interrogés, interdit en revanche toute conclusion sur l'articulation des processus de catégorisation de la couleur et des mécanismes langagiers. En effet, dans la mesure où il ne s'agit plus du phénomène couleur dans son état « naturel », c'est-à-dire ordinaire, porté par des objets, mais d'un artéfact qui ne le représenterait « véritablement » qu'en rapport à une conception de la couleur, on peut s'interroger sur l'interprétation que donnent des locuteurs qui ne sont pas aussi familiarisés que les expérimentateurs avec une certaine culture de l'abstraction et de la représentation de la couleur qui est la nôtre. Ainsi, sans aucune hypothèse sur la signification donnée par le sujet à ce matériel, les niveaux d'abstraction du terme ou du concept de l'informateur sont juste évalués dans leurs adéquations ou décalages à ceux de l'expérimentateur, concrétisés de manière exemplaire dans le stimulus proposé. ${ }^{12}$

11 Nous reprenons le terme « complex » employé par Berlin et Kay.

12 On a pu noter par ailleurs (pour une analyse détaillée voir Dubois \& Cance 2009, déjà cité) que les informateurs sont pour la grande majorité des locuteurs bilingues (leur langue maternelle 


\subsection{Une procédure expérimentale?}

Afin d'établir « scientifiquement » que la relation des termes de base aux couleurs de base est universelle, Berlin \& Kay vont introduire dans leur démarche linguistique et ethnographique une composante expérimentale, procédure qui sera largement réutilisée, en particulier dans les travaux ultérieurs de Rosch mais aussi dans le programme du World Color Survey initié par Berlin et Kay, $1969^{13}$ et actuellement généralisé à d'autres modalités sensorielles (voir le travail réalisé au Max Planck Institute de Nimègue et synthétisé dans Majid \& Levinson 2011).

2.3.1 Des listes de mots, des textes ou des discours?

La première étape du protocole expérimental - l'élicitation des termes de base reprend une procédure canonique en psychologie cognitive largement utilisée dans le domaine de la catégorisation qui consiste à demander à des sujets (locuteurs natifs) de produire une liste de mots, à partir d'un mot inducteur qui est un terme générique catégoriel (meubles, fleurs...). Dans le cas présent de la couleur, l'ouvrage de référence de Berlin \& Kay ne prend pas soin de citer les consignes données aux sujets (informateurs). Ils mentionnent simplement le fait que «no informant was ask to map his color terms until the investigation has elicited verbally his full list ${ }^{14}$ of basic color terms », les productions verbales des sujets étant par ailleurs filtrées selon les a priori des chercheurs relativement à la définition des termes de base (cf. plus haut). S'il s'agit de la «full list » de termes de base, il ne s'agit cependant pas d'un inventaire de tous les procédés langagiers disponibles dans une langue et utilisés par des locuteurs pour rendre compte de la description des phénomènes colorés, mais de formes lexicales simples dont le statut linguistique est par ailleurs non spécifié.

2.3.2. Des étiquettes sur des objets du monde?

Dans une seconde étape, la tâche qui est demandée au sujet est « d'apparier ses termes de couleur sur un tableau de pastilles de couleurs ». Le tableau stimulus (repris du nuancier Munsell) est recouvert d'un film transparent et on demande à l'informateur, à qui on donne un crayon-feutre noir, d'indiquer pour chaque terme de couleur X :

«1) toutes les pastilles qu'il appellerait, quelle que soit la condition, $X$,

2) le meilleur, l'exemplaire le plus typique de $X »($ Berlin \& Kay 1969, p. 7).

Outre le statut particulier (et restrictif) des unités lexicales retenues, les termes de base, une autre difficulté réside dans les restrictions portées à leurs conditions d'emploi en référence à une pastille de couleur. Nous nous trouvons devant une conception du langage qui exclut qu'on puisse, sous certaines conditions, désigner un objet à l'aide d'un terme $\mathrm{X}$ et sous d'autres conditions ce même objet à l'aide

et l'anglais) vivant en Californie. Lorsqu'aucun informateur n'est disponible, les listes de termes sont empruntées aux grammaires descriptives élaborées par les missionnaires. On comprendra bien ici que le partage au moins partiel du cadre de référence (linguistique et culturel) joue évidemment un rôle important dans les résultats de l'enquête.

13 Voir également voir MacLaury, 1997 ; et pour un bilan actuel : Kay, Berlin, Maffi, Merrifield, Cook. The World Color Survey. Stanford, CSLI, July 2009. http://www.icsi.berkeley.edu/wcs/.

14 C'est nous qui soulignons. 
d'un terme $\mathrm{Y}$, et donc que le terme de base devra répondre à l'exigence d'être le mot juste sinon vrai (le « veridical label », cf. Dubois \& Rouby (2002) pour les odeurs), pour désigner la ou les pastilles de couleur qui, elles aussi, représentent vraiment une catégorie de couleur sur le nuancier.

La procédure expérimentale qui prétend à être gage de scientificité s'appuie donc sur des a priori référentialistes qui simplifient l'équation de la relation des mots aux choses, en escamotant la définition des termes de base comme catégorie de langue, ainsi que la question de la matérialité de la couleur en laissant croire que chaque pastille réfère exactement à une couleur comme entité du monde et que les informateurs adhèrent d'évidence à cette conception.

Dans cette conception strictement référentialiste le langage est compris comme une structure d'appréhension d'une réalité intrinsèquement déterminée. La langue, comme ensemble de signes, est censée, selon les propres termes de Berlin et Kay, « encoder» les catégories de couleur qui donc sont préalables à la dénomination, et ce indifféremment, qu'elles soient dans le monde ou représentées par des pastilles. En d'autres termes, cette conception de la sémantique qui ne problématise pas même la notion de mot, ni celle de nom comme catégorie lexicale, mais demeure dans la tradition d'une sémantique du signe et relègue à la pragmatique l'étude des contextes et des idiosyncrasies d'usage, condamne la sémantique à un exercice d' « accurate denotation » (Lucy 1997, p. 322-323). Elle ne prend en considération que les recouvrements, sur plusieurs langues, de la dénotation de termes, sans tenir compte ni de son étendue référentielle, ni de sa distribution formelle c'est-àdire de sa position parmi les catégories de la langue avec lesquelles il se combine et se contraste, ni des usages différentiels selon les locuteurs, les pratiques et les situations de communication.

En effet, lorsqu'on implique des sujets dans cette tâche de mise en relation de termes avec des pastilles de couleurs en réponse à des consignes, cette tâche évidente, sans doute dans notre culture (en particulier pour les étudiants des universités occidentales) ne peut faire sens que sous les conditions suivantes :

- les consignes s'inscrivent dans un contrat de communication dans lequel il serait nécessaire de s'assurer que les divers acteurs impliqués interprètent la situation et sa finalité de manière convergente. Cela suppose que les sujets ont la même conception de l'unité lexicale que l'expérimentateur, qu'il puisse exister dans leur activité langagière la possibilité de faire fonctionner un élément de discours « under any condition »;

- en particulier qu'il puisse y avoir adéquation entre cet élément identifié comme objet de langue (par le linguiste mais peut-être pas par le sujet en question) et un élément de l'objet technique présenté ;

- et donc que les sujets conçoivent qu'une pastille (ou un ensemble de pastilles) puisse être directement associée à un de ces termes qu'on lui a fait produire.

\subsection{Lexique et catégories sémantiques : du naturel au situé}

La démonstration de l'universalité des catégories de couleur repose principalement sur l'utilisation d'une procédure de questionnement, qui si elle est universellement utilisée l'est davantage pour des raisons institutionnelles que pour sa validité 
scientifique. Elle repose en effet sur des théories largement partagées, explicitement, d'une part, dans les sciences du langage (Auroux 1992) mais aussi implicitement dans la théorie sémantique de sens commun dans notre culture et reprise dans l'interdisciplinarité confuse des sciences cognitives. En particulier la naturalisation du cognitif dans sa polysémie et son application indifférenciée aux mots, aux choses et aux catégories, conduit la linguistique cognitive à amalgamer la multiplicité des plans (psychologiques, linguistiques, anthropologiques, sociologiques) où se déploient les représentations et conceptualisations des couleurs : « La sémantique cognitive prend pour principe que le sens linguistique consiste en représentations ou processus mentaux, ce qui la conduit à s'absorber soit dans la psychologie, soit dans une philosophie spontanée » (Rastier 1993, p. 153) (voir également Dubois 2002, Resche-Rigon 2009 ainsi que Guignard 2011 sur ce point).

Le dépliement des différents plans d'analyse tant dans une sémantique des langues que dans une théorie cognitive de la catégorisation «située » dans les pratiques va maintenant nous permettre de construire un cadre interprétatif pluridisciplinaire qui respecte la constitution des objets spécifiques de chaque discipline, avant de spécifier leur articulation dans une théorie sémantique cognitive unifiée.

\section{D'AUTRES NUANCIERS : LES COULEURS EN PRATIQUES}

Nous venons de discuter des a priori implémentés dans la procédure de questionnement de Berlin \& Kay sur le terrain indifférencié de la linguistique et de la psychologie, cette indifférenciation ayant facilité leur démonstration de l'universalité des rapports au monde indépendamment des langues et des cultures. Ceci nous a permis de montrer les limites non pas de l'étude de formes lexicales isolées en tant que telles, ni d'une approche expérimentale en soi, mais de l'absence de prise en compte de ces productions langagières, des pastilles comme matérialisation particulière de la couleur, et de la procédure, cette fois dans une perspective de cognition « située ».

\subsection{Des nuanciers...}

En effet, le Nuancier Munsell s'inscrit dans une expérimentation qui en voulant respecter la contrainte (ou règle ?) d'un toute-chose-égale-par-ailleurs sauf la variable testée, à savoir l'universalité des catégories de couleurs malgré les variations culturelles et lexicales, rate sa cible. On montrera, à travers une diversité de nuanciers qui ressemblent au nuancier Munsell utilisé par Berlin \& Kay, mais qui ont été produits pour d'autres finalités que la reproduction exacte de la couleur, que le statut que l'on peut attribuer aux pastilles de couleurs et aux couleurs qu'elles représentent, n'est pas univoque mais dépend précisément des finalités de la couleur et de son implication dans la diversité des pratiques considérées.

Nous avons ainsi recueilli une série de nuanciers, artéfacts délibérément impliqués dans de multiples pratiques, et conçus pour promouvoir différents types de produits colorants : peintures pour artistes (peintures à l'huile, aquarelles), peintures décoratives pour la maison, colorants comme vernis à ongles, teintures 
de cheveux, carrosseries de voitures, laines, etc. Ces nuanciers, unifiés dans leur ressemblance au nuancier Munsell, diffèrent cependant quant à la nature des produits auxquels ils renvoient, et s'inscrivent donc dans des pratiques diversifiées qui concernent différentes personnes explicitement «ciblées » par les marques dans leurs spécificités socioculturelles. Il est notable que, sur chacun de ces nuanciers, à chaque pastille est régulièrement associée une «étiquette verbale » dont l'ensemble constitue ainsi pour nous un corpus linguistique attesté, formellement comparable aux données provoquées recueillies par questionnement de locuteurs natifs selon la procédure de Berlin et Kay.

\subsection{Les mots associés aux pastilles : des corpus}

Nous avons donc été amenées à considérer chaque forme lexicale associée à chaque pastille, non seulement comme la dénomination de la couleur représentée sur le nuancier, mais, suivant ce que nous mentionnions au paragraphe précédent, comme un élément d'un corpus d'un genre particulier, une liste de formes assurant la dénomination ${ }^{15}$ de chaque pastille, dans un discours que l'on peut qualifier de paradigmatique. ${ }^{16}$ Considérer ces listes comme un discours ou texte d'un genre particulier implique que la signification de chaque élément s'inscrive, de manière différentielle et non plus seulement référentielle, dans la diversité des pratiques sociales de communication (commercialisation) qui permettent la construction des nuanciers. Ces discours s'adressent soit à des « experts » praticiens qui partagent les connaissances qui ont présidé à l'élaboration et aux finalités du nuancier, soit aux acheteurs potentiels ou « cibles ».

\subsection{Voir et dire « rouge »?}

On prendra comme exemples particulièrement représentatifs la diversité des dénominations de la couleur (ou des couleurs ?) rouge(s) repérées sur différents nuanciers. Nous ne retenons ici que les pastilles qui sont désignées de rouge, rouge violet, rouge brique, rouge foncé, ou marron, par des locuteurs ordinaires lors d'un

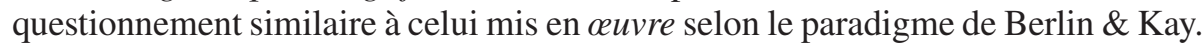
Voici les dénominations que nous avons pu relever dans les différents nuanciers sélectionnés (voir Dubois \& Grinevald 2003, pour une analyse détaillée).

1) Les peintures pour artistes (aquarelles, peintures à l'huile) utilisent des dénominations telles que : rouge vermillon français, laque garance rose, ou rouge de cadmium, rouge de Venise, laque carminée). Ce sont des dénominations techniques qui contrastent avec les dénominations des « mêmes » peintures grand public dont la finalité n'est pas d'utiliser la peinture comme couleur en soi mais pour produire des effets pour la décoration de la maison. Ainsi : rouge, rouge d'orient, grenadine, poterie, rouge basque ou paprika, ou encore brun van Dijk.

15 On considère les dénominations comme le résultat de processus de régulation sociale (normée) d'appariement des ressources d'une langue à des objets du monde qui s'en trouvent ainsi instaurés comme réalités mondaines, à la différence de la désignation qui caractérise des processus de référenciation individuels et contextualisés en discours.

16 Dubois \& Poitou (2002) sur les listes dans la recherche cognitive. 
2) Les cosmétiques, vernis à ongles et rouges à lèvres, utilisent également un lexique ordinaire qui se différencie cependant selon les (images des) marques :

Yves Rocher : Velours, Orient, Grenade

L'Oréal : ardent, incandescent, défendu, j'adore, Venise, Amour, comète

Agnès B. : brugnon, ethnique, comète, cœur, tulipe, flirt, Marilyn

3) Pour les teintures de cheveux, même si le registre des variations chromatiques est limité (en particulier pour le rouge $!^{17}$ ), on note, comme précédemment, un contraste entre les dénominations selon les marques de produits mais aussi entre les produits pour professionnels de la coiffure et entre les publics (consommateurs) « ciblés » : jeunes vs clientèle plus classique.

Professionnels (Schwarzkopf) : blond foncé acajou cuivré

«Ciblé jeune » (L'Oréal) : copper fusion

Ces données attestent ainsi, même sur un exemple restreint, d'une grande diversité des ressources linguistiques de la langue et la culture (françaises en l'occurrence) utilisées pour apparier une forme lexicale à une pastille de couleur, dès lors que l'on prend en compte la diversité des pratiques de la couleur dans notre culture et des usages de la langue (ici française) pour y référer.

\subsection{Une diversité d'objets linguistiques}

À partir de ces quelques données (dont une analyse plus détaillée peut être trouvée dans Dubois et Grinevald, déjà cité), on peut mettre en évidence différents types de ressources et de processus linguistiques.

3.4.1 Des mots : des noms ? des termes ?

D'abord une diversité de ressources lexicales qui couvre différentes catégories syntaxiques traditionnelles :

- on peut repérer en effet des adjectifs, non seulement des adjectifs considérés comme des adjectifs spécifiques de couleur (rouge, rouge vermillon, carminé) mais aussi des adjectifs référant à des objets ou concepts dont une des propriétés peut renvoyer à une couleur (ardent, incandescent, ethnique) ou un jugement (défendu), en particulier des formes adjectivales morphologiquement construites sur des noms (doré, cuivré), ou sur des verbes comme éclaircissant ;

- des noms utilisés comme adjectifs ${ }^{18}$ pour spécifier la couleur (de base), un certain type de rouge (brun Van Dijk) ;

- des noms d'objets directement utilisés comme noms de couleur typique d'un objet de référence : grenadine, poterie, cœur, tulipe (comme on l'observe régulièrement pour les odeurs, cf. Dubois 2000 et 2007);

17 « Rouge » est d'ailleurs exclu pour les cheveux ou utilisé de manière péjorative et « roux » n'est pas reconnu comme terme de base, puisqu'il s'applique sélectivement au domaine des cheveux, comme « châtain », témoignant ainsi de l'absence de fondement linguistique au concept de terme de base.

18 Procédé linguistique repéré depuis quelque temps déjà mais que nous voyons maintenant émerger dans le domaine olfactif avec le développement des odorants de synthèse et la dissociation de l'odeur de la source odorante (cf. David et al. 1997, Dubois \& Rouby 2002). 
- des termes techniques, spécifiques d'un domaine ou d'une communauté (laque garance rose, rouge de cadmium), renvoyant, dans ce cas précis, à la matérialité de la couleur comme pigment ;

- aussi des noms propres (Venise, Marilyn), des verbes (j'adore).

En bref, ce recueil de formes attestées dans ces nuanciers pour désigner des pastilles de couleur se déploie largement au delà des simples « termes de base », tant sur le plan des catégories syntaxiques que du statut lexical de ces formes, comme simple mot d'usage courant (poterie) ou comme terme d'un langage de spécialité. ${ }^{19}$

3.4.2. Des textes et des usages

En second lieu, il est notable que ces ressources lexicales se distribuent de manière différentielle selon les nuanciers, que l'on peut considérer, à la suite de Rastier (2001), comme des genres textuels, genres qui varient selon :

- les objets de référence (les « produits ») ;

- les locuteurs visés (ici les consommateurs « ciblés »);

- les marques (produisant des « images»).

Bref la dénomination des pastilles de couleur des nuanciers constitue un domaine où les professionnels du marketing s'avèrent gérer de manière créative et sereine une théorie sémantique où la fonction de communication du lexique contribue de manière essentielle à la référenciation, libérée d'une exigence de vérité référentielle (Mondada \& Dubois 1995).

En effet, ces objets, les nuanciers, sont des objets de communication qui répondent à des usages normés du vocabulaire, dont le souci n'est peut être pas l'exacte fonction référentielle (vériconditionnelle) mais davantage de produire des effets sémantiques suffisants pour constituer une description référentiellement valide et suggestive pour un comportement d'achat clairement différencié en fonction des catégories d'acheteurs (au sein d'une même (?) culture et, en tout cas, d'une même langue). Il nous semble dès lors étrange que les recherches interculturelles en linguistique cognitive poursuivent l'illusion de croire que le " même » nuancier Munsell puisse avoir la même signification et susciter de manière univoque des dénominations « vraies » de manière indifférenciée à l'intérieur d'une langue comme au sein des différentes langues et cultures étudiées.

\subsection{Inférences cognitives : lexique, concepts et pratiques}

À partir de ces analyses linguistiques, qui relèvent tant de la grammaire traditionnelle que d'une linguistique des discours et textes, il est maintenant possible d'envisager, dans la visée d'une contribution à une sémantique cognitive, quelles inférences peuvent être émises et quels statuts accorder quant aux réalités cognitives auxquelles réfèrent la diversité de ces modes de dénomination. En nous appuyant sur les recherches psychologiques relatives aux catégories perceptives dans différentes modalités sensorielles (déjà exposées de manière plus détaillée

19 Sur les différences entre mot et terme, et usage courant vs usage terminologique des formes lexicales en analyse sensorielle, voir Giboreau et al. (2007). 
dans Dubois 2000, 2007 et 2009), on insistera plus particulièrement sur la nécessité de distinguer les connaissances individuelles construites à partir du rapport sensible (et solitaire) au monde ${ }^{20}$ et les connaissances partagées acquises, en particulier à partir de pratiques collectives et de l'usage des langues communes ou de spécialité qui se manifestent dans la dénomination des pastilles des nuanciers.

On peut en effet tenter d'associer à ces divers modes d'expression en langue et types de textes ainsi différenciés par leurs finalités, différents statuts sémiotiques de la couleur (ou des couleurs), autres que la fonction référentielle qui relie un mot simple (un nom, un terme) à une entité du monde ; en particulier celle d'indice de l'existence de « quelque chose du monde » (Eco 1976). En tant qu'indice, la (les) couleur(s) peuvent se trouver diversement sémiotisées, et en particulier être plus ou moins conceptuellement autonomisées ou détachées des objets ou sources qui, dans le monde « réel $»^{21}$ suscitent les sensations.

Ainsi, de la diversité des dénominations couplées à celle des pratiques de la couleur qui se manifeste dans les nuanciers, on peut identifier diverses conceptualisations de la couleur, que nous pouvons systématiser selon quatre registres :

- les couleurs comme matérialités du monde, comme pigment, la couleur se trouvant alors constituée comme matière colorée et/ou colorante, intégrant d'autres propriétés indissociables de l'expérience sensible des pigments, telle qu'elle «fonctionne » dans la terminologie des peintures pour artistes (rouge de cadmium véritable);

- les couleurs comme indices sémiotiques de l'existence de « quelque chose d'autre dans le monde » qui se donne ainsi à voir, qui donne le nom à la couleur (bleu canard, vert pomme) dans la mesure où elle est propriété caractéristique de l'objet (poterie, sable, mais aussi orange, étrangement retenu comme terme de base...). La couleur n'est alors pas (pas plus que les odeurs ou les bruits) autonomisée, n'a pas pris son indépendance (y compris lexicale) du support ou de la source dont elle est une caractéristique identitaire, comme c'est le cas pour les couleurs de peintures décoratives, et autres produits de large consommation ;

- les couleurs comme apparences « posées » sur les choses, qui résultent de la longue tradition des pratiques de la coloration et des teintures, jusqu'au développement plus récent de la chimie des colorants et de la coloration de produits de plus en plus

20 Nous n'utilisons pas à dessein le terme de perception, car ce thème est lui-même objet de débats quant au caractère strictement individuel (subjectif !) contraint par des déterminations physiologiques et/ou partiellement influencé par des connaissances préalables. Cette question de la co-saisie du sens dans l'acte de perception est un problème qui s'est trouvé diversement abordé en philosophie (Voir notamment Merleau Ponty (1934) : «La conscience percevante n'est pas une conscience pure car percevoir, c'est percevoir du sens »; et Cassirer (1953, p. 19) : «Il ne faut point partir de l'objet comme s'il nous était immédiatement connu mais bien des lois de la connaissance, qui seules nous sont véritablement accessibles et comportent dès l'abord quelque garantie »).

21 Le privilège accordé aux situations expérimentales en psychologie, conduit en effet à oublier que les stimuli proposés aux « sujets » en laboratoire (comme d'ailleurs les pastilles de couleurs) sont des artéfacts qui ont techniquement «abstrait » certaines propriétés du « monde réel », telles que les qualités colorées ou acoustiques, alors que tout individu en situation «normale » quotidienne ne perçoit que des objets colorés ou sonores. 
diversifiés pour les visées de commercialisation (blond très clair éclaircissant, pour les teintures de cheveux pour professionnels, par exemple);

- enfin, les couleurs comme effets produits. Ce n'est plus une couleur comme telle qui est désignée mais sa valeur symbolique, cette fois techniquement autonomisée des objets qui sont colorés. La dénomination de la couleur, libérée de la stricte exigence référentielle vraie, est soumise alors à d'autres déterminations, et donne libre cours à la créativité des politiques de marketing, afin de susciter l'achat des produits (comme c'est le cas pour les couleurs de teintures de cheveux « ciblées jeunes »(Cooper fusion, Hollywood, Tahiti...)), les couleurs de peintures de voitures (Santorin, Pharaon), les rouges à lèvres ou vernis à ongles (flirt, Marilyn, Hot Ginger, Red Fiction), etc. (voir Dubois \& Grinevald, déjà cité mais également Wyler 2007 pour des analyses et exemples plus détaillés).

À travers ces nuanciers qui constituent des terminologies, et en ce sens des réponses collectives à la procédure d'appariement des mots aux choses (pastilles de couleur) classiquement utilisée à l'échelle universelle par le programme World Wide Color Survey, on est ainsi amenées à considérer que le succès de la procédure de Berlin et Kay serait alors dû :

1) aux contraintes imposées par le mode opératoire mis en œuvre ;

2) à l'analyse des réponses obtenues :

- s'il s'agit d'une forme simple, que celle-ci pourra être candidate à devenir un terme de base selon des critères fondés sur les a priori des chercheurs et par ailleurs discutables ;

- s'il s'agit d'un énoncé complexe, les chercheurs concluent que les locuteurs n'ont pas de terme pour désigner les couleurs considérées et donc que leur langue présente un «lexical gap ».

Ces procédures de questionnement et de traitement des données relèvent implicitement :

- soit d'une conception adamique (donc primitive ou naïve) de la sémantique lexicale, consistant à croire que les mots sont des étiquettes posées sur des choses préexistantes (ontologies) ;

- soit d'une soumission à l'autorité des sciences de la nature qui consacrent et officialisent certains mots comme termes de couleur.

Cette analyse cognitive est à relier à la fois :

- aux recherches linguistiques en diachronie telles celles sur des termes de couleurs dans les langues romanes (André 1949, Kristol 1978) ou en synchronie sur d'autres langues et d'autres cultures (Conklin 1964, Guedou \& Coninckx 1986, Lucy 1997, Evans \& Levinson 2009 parmi de nombreux autres) qui apportent d'autres arguments qui rendent également problématique le concept de terme de base ;

- ainsi qu'aux problématiques développées par Latour que nous rejoignons dans son analyse des dispositifs techniques ou expérimentaux, comme artéfacts inscrits dans des pratiques et des cultures et donc diversement intériorisés (et interprétés voir interprétables) par les sujets interrogés. 
Après avoir revisité le protocole de dénomination de pastilles de couleur au sein de nuanciers inscrits dans des pratiques, il s'agira maintenant de s'intéresser à l'inscription des couleurs dans des discours d'évaluation de la qualité visuelle d'espaces réels et visuellement simulés, constituant des dispositifs.

\section{COULEURS EN PRATIQUES ET EN DISCOURS}

\subsection{Des discours de l'expérience individuelle de la couleur}

Ce deuxième exemple de recherches empiriques s'intéresse également aux ressources lexicales disponibles en langue française pour faire référence au phénomène de la couleur mais cette fois situées dans un autre type de discours et de pratiques. En effet notre argumentation s'appuie sur l'analyse de discours collectés dans un cadre expérimental d'évaluation de la qualité visuelle d'espaces complexes particuliers, ici des habitacles automobiles. ${ }^{22} \mathrm{~A}$ la différence des listes de dénominations spécifiques de couleur accompagnant divers types de nuanciers commerciaux (peintures, cosmétiques...), que l'on peut qualifier de terminologies d'experts collectivement négociées et consensuelles au sein des communautés de pratique respectives, les discours ici considérés rendent compte de l'expérience individuelle que quelques locuteurs (non experts du domaine automobile) tentent d'expliciter et de partager en discours avec leur interlocuteur. ${ }^{23}$ Ce qui relève du consensuel et partagé dans ces conditions ce sont justement les ressources linguistiques et les processus discursifs à disposition des locuteurs pour faire part de leur expérience individuelle de la couleur.

Il s'agit de prêter une attention particulière sur le plan linguistique, à la diversité des modes d'inscription syntaxique des formes lexicales de couleur et sur le plan psychologique, aux inférences cognitives quant à la diversité de conceptualisations de la couleur qui peuvent être tirées de cette diversité de procédés syntaxiques, dans le prolongement des analyses réalisées dans la section 3.

\subsection{Un dispositif expérimental : des matérialités à évaluer}

Les nuanciers de couleur qui constituent la base du matériel expérimental dans les travaux en sémantique des couleurs offrent tous le même type de matérialité ou plutôt de re-matérialisation (représentation matérielle) de la couleur étroitement tributaire des développements technologiques comme tout capteur ou instrument de mesure des phénomènes physiques perceptibles. ${ }^{24}$ Dans la section 3 , il s'est agi

22 Ce travail de recherche a été effectué dans le cadre d'une thèse CIFRE impliquant l'Université Paris 3 - Sorbone Nouvelle, PSA Peugeot Citroën et le LCPE (Cance 2008a).

23 Ici la personne qui a réalisé les entretiens avec les sujets, N. Cavelier, que nous remercions pour sa précieuse aide dans la constitution et la passation du protocole ainsi que pour ses analyses (Cavelier 2003).

24 À ce titre ils constituent, comme dit précédemment, des artéfacts, re-matérialisant des connaissances, au même titre qu'un thermomètre ou un microscope. 
de contraster différents nuanciers relevant de plusieurs expertises de la couleur, révélées par des dénominations spécifiques pour chacune d'entre elles.

Dans le cas présent, une analyse différentielle de discours est rendue possible par le dispositif expérimental de questionnement mis en place qui offre aux locuteurs différents types de matérialité (de l'habitacle automobile et par extension des couleurs de ces habitacles) à évaluer.

L'étude a en effet consisté à réaliser 15 entretiens semi-directifs dans 3 dispositifs de présentation de «mêmes » habitacles ${ }^{25}$ :

- Le dispositif $2 D$, un écran Plasma®, propose aux locuteurs des images statiques bidimensionnelles.

- Dans le dispositif $3 D$, le locuteur est assis dans un siège conducteur et porte des lunettes stéréoscopiques avec capteur de mouvement. Des images tridimensionnelles projetées sur quatre parois (2 latérales, 1 frontale, 1 au sol) d'une salle cubique (CAVE®) lui donnent l'illusion d'être à l'intérieur d'un véhicule.

- Le dispositif REEL consiste en deux véhicules à l'arrêt dans un hangar et à l'intérieur desquels se fait le questionnement, l'interviewé étant assis sur le siège conducteur, « comme » dans le système de simulation 3D.

Les personnes interviewées ont successivement été confrontées aux trois dispositifs (dans lesquels leur étaient présentées différentes ambiances d'habitacle). L'ensemble des commentaires d'évaluation produits dans chacun des dispositifs a donné lieu à la constitution de 3 corpus $^{26}:$ corpus 2D, 3D et Réel.

Alors que le nuancier de pastilles de couleurs est le seul niveau de représentation et l'unique matérialité proposée, nous avons ici à disposition différentes formes de matérialisation de connaissances et d'abstraction (d'une voiture « réelle » / d'un espace holisensoriel « virtuel » qui la représente). En nous référant de nouveau au travail de Latour (déjà cité), on pourrait dire que si le nuancier est le résultat d'un processus complexe et progressif d'abstractions successives, ce dispositif expérimental nous met en présence de différentes étapes : la voiture comme terrain empirique et les simulations $2 \mathrm{D}$ et $3 \mathrm{D}$ (re-)construites à partir d'abstractions successives $^{27}$.

Ces dispositifs permettent ainsi d'évaluer l'équivalence perceptive ou la non adéquation entre les différents modes de présentation et par là même contribuent à la réflexion sur la validité écologique des protocoles expérimentaux en sciences humaines contemporaines (introduite par Brunswik 1956 puis Gibson 1979 et développée dans la modalité sonore par Guastavino 2005, 2009 et dans la modalité visuelle dans Cance 2009).

25 Pour une description complète du protocole expérimental, cf. Cance 2008a et 2009.

26 Notre définition de corpus dans le cas présent ne répond notamment pas aux critères quantitatifs en usage en linguistique de corpus, cependant nous conservons cette qualification puisqu'il s'agit ici de données empiriques attestées, recueillies et constituées en tant qu'observables que nous soumettons à l'analyse.

27 Dans un même temps, c'est aussi l'inverse puisque la voiture comme artéfact repose sur ces étapes progressives de design avant d'être réalisée. Et les simulations sont produites à partir du modèle CAO (Conception Assistée par Ordinateur) qui a permis de réaliser le véhicule... 
Cela nous permet également de tester si ces différents dispositifs suscitent différentes expériences sensorielles et conceptualisations d'un objet hypothétiquement identique, et d'élargir notre raisonnement à différents types de matérialité identifiables à travers différents types de discours (caractérisés par des processus syntaxiques et discursifs différents).

\subsection{Inscriptions syntaxiques et conceptualisations de la couleur}

Un relevé systématique de toutes les formes lexicales de couleur présentes dans les 3 corpus (2D, 2D et Réel) a permis d'identifier des formes dites génériques (couleur, coloris, ton, teinte, tonalité, nuance) et des formes référant à une couleur spécifique (noir, gris, beige, bleu, rouge, vert...). Une analyse des formes spécifiques les plus utilisées $^{28}$ (beige, gris et noir) a mis en évidence des inscriptions syntaxiques différentielles en tant que formes nominales vs adjectivales et parmi ces dernières en tant que formes attributives ou épithètes selon la couleur référée et la matérialité de l'expérience offerte par le dispositif de présentation (pour une analyse détaillée voir Cance 2008a et 2008b).

Ces analyses offrent un contre-argument au postulat que les termes de couleur ont un sens unique et indépendant des situations de référence auxquelles ils s'appliquent et de leur inscription en discours. La catégorie syntaxique des mots de couleur se révèle un indicateur linguistique pertinent pour identifier différentes conceptualisations de la couleur :

- Beige, majoritairement employé comme substantif simple précédé d'un déterminant défini (le beige) ou accompagné d'un adjectif épithète (beige rosé), est davantage construit en discours comme une entité du monde que les locuteurs peuvent évaluer.

- Gris employé tantôt comme substantif et tantôt comme adjectif renvoie respectivement davantage à la construction cognitive du gris conçu soit comme entité du monde plus (un gris) ou moins autonome (le gris du plastique), soit comme propriété d'un objet (la voiture grise) ou d'une couleur (des tons gris).

- Noir employé majoritairement comme adjectif renvoie à une couleur comme propriété d'une matière ou d'un élément (un bouton noir).

Le contraste entre l'adjectif beige presque toujours épithète et les adjectifs gris et noir le plus souvent attributs et modalisés par différents adverbes contribue également à différencier les statuts cognitifs de ces différentes couleurs :

- Beige réfère à une propriété inhérente à l'objet qualifié, alors que gris et noir renvoient à une dimension « quantifiable» (hyper gris...) et contribuent à construire la référence en discours (bah là la radio c'est toujours gris).

- La couleur comme propriété de la matière désignée par les constructions de type Nom de matière+Adjectif de couleur (le tissu noir) contraste avec la couleur comme indice d'une matière dans des constructions de type Nom de couleur+Nom de matière (le gris métal, le beige plutôt cuir) qui concernent davantage les substantifs gris et beige.

28 En lien direct avec les ambiances colorées des habitacles automobiles présentés aux locuteurs. 
Cette analyse révèle des variations syntaxiques couplées à des variations sémantiques d'une «même » forme lexicale de couleur inscrite dans des discours d'évaluation de la qualité visuelle. Selon qu'elle sera employée comme nom, adjectif épithète ou attribut, elle n'a pas le même sens et ainsi ne réfère donc pas à une «même » couleur ou tout au moins à une même conceptualisation d'un phénomène considéré sur des paramètres physiques. Une même forme lexicale, selon son inscription syntaxique, ne renvoie pas à une seule construction cognitive, mais à des processus sémiotiques différents qui jouent différents rôles dans la construction globale de l'expérience visuelle.

\subsection{Constructions cognitives, dispositifs techniques et matérialités sensibles}

Les formes lexicales beige, gris et noir constituent tous trois de bons indicateurs des contrastes entre dispositifs. Ainsi les distributions différentes d'adjectifs de couleur épithètes et attributs dans les trois dispositifs constituent un premier indice linguistique d'une relation différenciée au type de matérialité (re-présentation) proposé aux locuteurs.

- Le corpus 2D se caractérise par la présence plus importante d'adjectifs de couleurs épithètes ${ }^{29}$ inscrits dans des syntagmes nominaux permettant la mise à distance de l'image explorée, décrite et ainsi recomposée. Ces adjectifs épithètes participent avec d'autres marqueurs et ressources (formes nominales abondantes, coordinations, le $N$ des couleurs) à rendre compte du dispositif 2D comme amenant les locuteurs à construire leur évaluation de manière globale avec un certain niveau d'abstraction, dans une pratique proche de la description picturale ou photographique ;

- Le corpus 3D suscite plus d'emplois de beige, noir et gris en tant qu'attributs. Devant ce dispositif non familier, dans lequel le partage de la référence perceptive avec leur interlocuteur n'est que partiel ${ }^{30}$, les locuteurs doivent construire la référence. Ils ont recours aux adjectifs de couleur désignant alors des propriétés qui permettent de reconstruire la matérialité des objets représentés visuellement à partir de la couleur, avant de pouvoir dans un second temps les évaluer.

De plus, une analyse de l'inscription en discours de beige, gris et noir permet également d'identifier des positionnements différents des sujets vis-à-vis des couleurs allant d'une évaluation « subjective » (la couleur pour moi) :

"-Je trouve que ça a un beau noir euh très uni et qui brille euh qui brille bien il est euh j'le trouve très beau. » (Corpus Réel),

à la description « objectivante » de la couleur (la couleur en soi) :

«-En haut il y a du noir et là i(l) y a du métal. » (Corpus 3D).

29 La majorité des analyses de corpus portant sur l'adjectif sont réalisées avec des corpus écrits, dans lesquels le fonctionnement syntaxique de l'adjectif est un peu différent. Une étude de Blasco-Dulbecco et al. (2004) portant sur les spécificités syntaxiques de l'adjectif à l'oral a mis en évidence le même type de contraste entre constructions épithètes et attributives.

30 Seuls les locuteurs portent les lunettes 3D à capteur de mouvement qui permettent une reconstruction en temps réel de l'image en fonction des mouvements de leur tête. 
Ces énoncés sont différemment répartis dans les trois corpus ${ }^{31}$, et différemment encore selon les couleurs. En résumé, les dispositifs de simulation suscitent davantage de descriptions via des procédés de construction discursive de la référence (41,2 et 51,4\% d'énoncés de construction) que le dispositif Réel (22\%), dans lequel les couleurs prennent surtout part à des processus d'évaluation (88\% d'énoncés d'évaluation).

Enfin, une analyse spécifique de ces énoncés descriptifs de construction de la référence produits au sein des dispositifs $2 \mathrm{D}$ et $3 \mathrm{D}$ a permis de mettre à jour le rôle structurant/d'étayage de beige, gris et noir qui peut varier suivant les dispositifs et les situations :

- les formes lexicales de couleur peuvent être utilisées comme «balises », points fixes autour desquels les locuteurs tentent d'identifier ou de reconstruire des couleurs qui leur échappent :

$$
\text { «-J'vois gris j'sais pas si c'est noir hein, j'vois gris euh. » (Corpus 3D). }
$$

Ces énoncés se caractérisent par de nombreux adjectifs de couleur attributs, marques d'incertitude et d'évidentialité (je ne sais pas, j'ai l'impression, je vois gris, je le vois presque noir). Principalement issus du corpus $3 \mathrm{D}$, ils révèlent un problème lié aux caractéristiques techniques de ce dispositif. ${ }^{32}$

- Les formes lexicales (beige principalement sous forme nominale suivi de gris comme nom ou adjectif) peuvent également être utilisées comme indices de la matière dans des situations de perception où les indices tactiles font défaut :

$$
\text { «-C'est vrai que p(eu)t-être le beige là, en l'occurrence, } i(l) \text { fait penser à du }
$$
cuir. »(Corpus 2D).

L'usage privilégié de noms de couleur atteste de cette nécessité de construire à travers le discours l'identité des matières supposées, lorsque les locuteurs sont face à des dispositifs de simulation visuelle.

- Enfin elles peuvent également servir d'étayages à la construction de la référence et de la matérialité d'objets et d'espaces « virtuels ».

On a ainsi pu repérer des procédures d'identification et de référenciation aux objets de l'habitacle (davantage caractéristiques du dispositif 3D) :

$$
\text { «-Je suppose que c'est le lecteur de CD qui est gris. » (Corpus 3D). }
$$

Mais à l'instar de l'identification d'une matière, la construction des objets dans les situations simulées via l'étayage des adjectifs de couleur attributs gris et noir n'est pas nécessairement une fin en soi pour les locuteurs. Alors que dans les habitacles réels, le rapport à l'espace se construit dans la confrontation multisensorielle avec les objets, dans les dispositifs $2 \mathrm{D}$ et 3D, ce sont les objets déjà construits et posés

31 Pour une analyse détaillée voir Cance et al. 2009 et Cance 2008b.

32 Par exemple, « créer » la couleur noire avec de la lumière, tout en jouant sur les contrastes pour gagner en précision d'image, s'avère une entreprise délicate, inscrite ici dans les discours des locuteurs qui repèrent l'ambiguïté, et une certaine indéfinition de la couleur présentée. Ceci vient confirmer des résultats lexicaux quantitatifs mettant en évidence une répartition alternée entre gris et noir dans les trois corpus : une majorité d'occurrences de gris dans le corpus 2D, une majorité de noir dans le corpus Réel et le corpus 3D comme intermédiaire (toujours davantage de gris mais moins qu'en 2D). 
en discours au moyen notamment des couleurs, qui vont contribuer à construire discursivement l'espace perçu visuellement. Ainsi :

"-Y a beaucoup de gris [...] le volant euh au dessus du enfin sous l(e) parebrise là tout est gris.» (Corpus 2D.)

\subsection{Lexique en discours : des pratiques discursives situées}

Sur un plan linguistique, l'analyse de ce corpus de discours sur la couleur a permis de mettre en évidence une diversité des modes d'inscription syntaxique des formes lexicales de la couleur en français que le travail à partir d'exemples ou de listes de termes ne permettrait pas. On a pu voir comment la référence à et par la couleur est diversement travaillée et négociée en discours en fonction de l'expérience individuelle et dans l'interaction collective (ici le partage de référence avec l'interlocuteur) (cf. Mondada \& Dubois 1995 mais aussi Clark \& WilkesGibbs 1986).

Ceci ouvre sur la nécessité et la richesse d'un travail en sémantique cognitive qui prendrait en compte ces discours « ordinaires » attestés de type conversation dont l'analyse permet de préciser les processus de co-construction dynamique des catégories linguistiques et cognitives (cf. Bieler 2011, dans le domaine du goût).

Dans ce cas précis, sur le plan cognitif l'analyse de l'inscription discursive des formes linguistiques de couleur au sein de commentaires descriptifs et évaluatifs d'ambiances d'habitacle permet de confirmer et de préciser l'identification de différentes conceptualisations des couleurs déjà mises en évidence dans la partie 3 :

- Les couleurs, en tant que connaissances culturellement, historiquement élaborées, peuvent être envisagées comme des entités autonomes ;

- elles peuvent également, comme critère d'évaluation, participer à l'appréciation visuelle globale et l'affecter ;

- en tant que propriétés visuelles d'une matière ou d'un objet, elles constituent également un indice pour l'identification et la construction de cette matière, de cet objet ou d'un espace, notamment lorsque des indices tactiles et kinesthésiques font défaut. Étayant la construction en discours de la matérialité de l'espace exploré, elles permettent alors de construire un cadre de référence pour l'évaluation.

D'un point de vue méthodologique, il nous semble intéressant de mettre en parallèle la situation (non familière) du dispositif 3D dans la culture occidentale, qui nous a permis de révéler des processus de construction «online » de la référence partagée et la situation de questionnement avec des pastilles de couleurs dans d'autres cultures. On peut en effet se demander dans quelle mesure des informateurs d'une culture n'ayant pas construit le même type de connaissances sur la couleur ne se trouvent pas face au Munsell comme nos locuteurs face au dispositif 3D : il s'agit davantage de construire la référence à cette nouvelle matérialité que de considérer le stimulus comme représentation adéquate DU réel. Mais l'évaluation de cette hypothèse ne sera possible qu'à la condition de s'affranchir de la restriction aux seuls termes de base et de considérer ces formes lexicales dans leur inscription discursive. 
Les couleurs sont donc envisagées comme des objets cognitifs co-construits par le discours, l'expérience sensible et les connaissances préalables, mais également utilisées comme étayages de la construction de l'espace (objectivé) en discours et comme support de l'évaluation. Ces différents statuts de la couleur tant en discours qu'en cognition, témoignent de la couleur comme d'un concept multiple et de pratiques de la couleur comme « matières à penser » et à construire le réel (Warnier 1999).

\section{CONCLUSION}

Les recherches en linguistique cognitive que nous venons de retracer s'inscrivent dans la continuité des problématiques initiées par les linguistes américains de la côte ouest des États-Unis. D'une part, nous pensons avoir apporté des éléments qui confirment ces affirmations à travers nos analyses de la dénomination des phénomènes colorés. D'autre part, nous avons également pu identifier certaines limites de cette conception.

Nous avons en effet montré la nécessité de tenir compte de la diversité des ressources linguistiques en œuvre dans la dénomination des couleurs, qu'elles soient contraintes par la matérialité de pastilles de couleur, ou utilisées pour rendre compte de la qualité d'un espace visuel auquel elles contribuent. Ces dénominations ne peuvent se limiter à l'usage de termes spécifiques de couleurs (a fortiori les seuls termes de base). De nombreuses formes lexicales sont susceptibles de rendre compte de l'expérience sensible des couleurs et ce différentiellement selon les pratiques de la couleur. En outre, la diversité des inscriptions syntaxiques des mots de couleurs en discours permet de repérer également d'autres modes de référenciation et de conceptualisation de la couleur, de la couleur comme sensation, comme effet, à la couleur perçue comme connaissance renvoyant à une objectivité du monde.

Ainsi, au lieu de fonder les catégories (lexicales) dans une ontologie, fût-elle «mentalisée » par l'expérience sensible, comme le prétend la conception naturaliste de la cognition dominante en linguistique cognitive, nos données suggèrent d'analyser la diversité des conceptualisations dans la complexité des processus linguistiques de dénomination au sein de pratiques (y compris de pratiques langagières). Une telle approche qui s'inscrit dans la perspective actuelle d'une « cognition située » impose de déplier la complexité des processus de dénomination et de conceptualisation quelque peu négligés dans une linguistique cognitive qui reste limitée à une conception référentielle du lexique comme signe. Cette conception, fut-elle médiatisée par des « catégories naturelles » tout aussi universelles que le concept inscrit dans la triade fondatrice de la sémantique (y compris cognitive) (Rastier 1990) et dont le statut linguistique ou psychologique n'est jamais clairement explicité, demeure dans la simplicité de la relation «mot-chose».

La diversité repérée dans les dénominations impose donc de prendre d'abord en compte les ressources différentielles des langues, en particulier la présence de formes lexicales à disposition des locuteurs, et d'en faire l'inventaire, ce qui est le cas actuellement non seulement pour les couleurs, mais aussi pour les 
odeurs, les bruits et les sons, les goûts ... (cf. Majid \& Levinson, déjà cité). Il est cependant dommage que cet inventaire reste conçu dans le cadre très restreint d'une conception du langage comme nomenclature et dans des procédures d'appariement des mots aux choses qui conduisent à des interprétations de la diversité en terme de «failure » ou de «lexical gap » en regard d'une langue de référence... généralement l'anglais.

Il est donc également nécessaire d'étudier les modes de référenciation des formes lexicales en discours, dans la diversité des langues, afin d'éviter à la sémantique cognitive de rester « objectiviste », et comme le remarque Paveau (2007) à la suite de Rastier, de considérer la diversité des langues comme variations à partir d'un point fixe qui correspond étrangement à « notre » représentation contemporaine du monde.

«Les formes nouvelles du cognitivisme, dont relève la sémantique cognitive, ne se réclament plus de ce rationalisme [du cognitivisme chomskyen], mais demeurent universalistes dans la mesure où elles réitèrent, nous le verrons, des gestes caractéristiques de la philosophie transcendantale.

L'unité de l'esprit humain n'étant généralement pas mise en doute, et les significations étant rapportées à des représentations ou des opérations mentales, personne ne formule l'hypothèse qu'il existe autant de sémantiques que de langues, soit six mille au bas mot. Or, le problème fondateur de la diversité des langues distingue décisivement la sémantique linguistique de la philosophie de la signification. La sémantique cognitive relève donc, pour l'essentiel, de la tradition philosophique : la recherche des primitives conceptuelles, universaux et archétypes cognitifs se situe à l'évidence dans cette tradition. » (Rastier 2005).

La recherche linguistique doit alors être couplée avec une théorie psychologique qui elle aussi se devrait de prendre en compte la diversité des connaissances élaborées dans la diversité des pratiques humaines, au lieu d'unifier le fonctionnement cognitif humain en terme d'information (Dubois 2002) en éliminant la diversité des connaissances construites à partir de processus de sémiotisation (connaissances communes, expertes, savoirs et savoir-faire, cf. Dubois 2008).

Enfin, au niveau des méthodes, cette orientation impose d'intégrer une approche analytique d'une linguistique de l'exemple, tout comme la démarche expérimentale d'élicitation de " réponses verbales », à des analyses de corpus attestés et effectivement produits dans le cadre de pratiques « ordinaires ». Suivant Achard-Bayle \& Paveau (2007), on peut en effet relever que « ce qui gêne dans l'actuel réseau des Coglingers (revue Cognitive Linguistics, colloques ICLC), c'est que les modèles centraux (de Lakoff, Turner \& Fauconnier) sont le plus souvent simplement illustrés par la diversité des langues, conçues comme des corpus d'application ».

C'est ainsi la diversité des ressources des langues et celle des pratiques langagières qui permettent de rendre compte à la fois de l'expérience sensible individuelle manifestée dans les discours de la subjectivité (Vion 2009) et de la construction de connaissances partagées à travers des pratiques communes, voire à l'établissement d'une objectivité telle qu'elle se manifeste dans le discours scientifique. 
C'est donc l'analyse linguistique de la diversité des modes de référenciation qui permet d'élaborer une théorie linguistique cognitive «située » où s'articulent une sémantique des langues et une théorie psychologique des catégories dans une sémiotique cognitive des cultures. Le risque est cependant de confondre les connaissances spécifiques des différents champs disciplinaires concernés (psychologie, sociologie, anthropologie, développement technologique et théorie scientifique des couleurs, histoire des sciences). Tous ces domaines se trouvent eux-mêmes constitués dans et par une pluralité de discours, tels que les explorent les recherches de van Dijk (1993) ou de Rastier (2001) par exemple ou plus près de nous Paveau (2007, Achard-Bayle \& Paveau, ce numéro) qui mettent les pratiques humaines au centre de la cognition, que cette dernière soit appréhendée comme construction individuelle ou collective et partagée (Suchman 1987, Hutchins 1995 et dans une tradition européeenne : Leroi-Gourhan 1964, Warnier 1999).

\section{RÉFÉRENCES BIBLIOGRAPHIQUES}

Achard-Bayle, Guy (2007). «Les réalités conceptuelles et leur ancrage matériel. Les sémantiques cognitives et la question de l'objectivisme », CORELA, numéro thématique Cognition, discours, contextes. [En ligne] http://corela.edel.univ-poitiers.fr/index. php?id=1532.

Achard-Bayle, Guy \& Paveau, Marie-Anne (2007). « Editorial », CORELA, numéro thématique Cognition, discours, contextes. [En ligne] http://corela.edel.univ-poitiers. fr/index.php?id=836.

André, Jacques (1949). Étude sur les termes de couleur dans la langue latine, Paris, Klincksieck.

Auroux, Sylvain (1992). « Théories linguistiques et opérations mentales », Histoire, Épistémologie, Langage 14, 1-13.

Berlin, Brent \& Kay, Paul (1969). Basic Color Terms: Their Universality and Evolution, Berkeley, University of California Press.

Bieler, Larissa (2011). « On Understanding Taste. Cooperative Strategies of Meaning Constitution in Conversations about Taste ». Symposium "Talking about Taste" "Sensory Semantics and the Language of Taste" (SenS), Zurich 15-16 avril 2011.

Blasco-Dulbecco, Mylène \& Cappeau, Paul (2004). "Quelques remarques sur l'adjectif à l'oral », François, Jacques (éd.), L'Adjectif en français et à travers les langues, Caen, Presses Universitaires de Caen, 413-428.

Brunswik, Egon (1956). «Perception and the Representative Design of Psychological Experiments », Berkeley, University of California Press.

Cadiot, Pierre \& Visetti, Yves-Marie (2001). Pour une théorie des formes sémantiques : motifs, profils, thèmes, Paris, PUF, Formes sémiotiques.

Cance, Caroline (2008a). Expériences de la couleur, ressources linguistiques et processus discursifs dans la construction d'un espace visuel : l'habitacle automobile. Thèse de doctorat de Sciences du Langage. Université de la Sorbonne Nouvelle - Paris III.

Cance, Caroline (2008b). Termes de couleur en discours et concepts de couleurs. Lidilem (eds). Autour des langues et du langage: Perspective pluridisciplinaire, Grenoble, Presses Universitaires de Grenoble, 161-168.

Cance, Caroline (2009). "Stimuli, Dispositifs expérimentaux et Mondes construits », Dubois, Danièle (éd.), Le sentir et le dire : concepts et méthodologies en linguistique et psychologie cognitive, Paris, L'Harmattan, 107-136.

Cance, Caroline, Giboreau, Agnès \& Dubois, Danièle (2009). Co-construction du sens et de l'espace en discours : l'exemple des couleurs. Havu, Eva, Härmä, Juhani, Helkkula, 
Mervi, Larjavaara, Meri \& Tuomarla, Ulla (eds), La langue en contexte, Helsinki, Société Néophilologique, 363-375. [En ligne] http://www.helsinki.fi/jarj/ufy/RSL.htm. Cassirer, Ernst (1953). La philosophie des formes symboliques, Paris, Éditions de Minuit.

Chomsky, Noam (1968). Language and Mind, New York, Harcourt (Trad. franç. (1970) Le langage et la pensée, Paris, Payot).

Cavelier, Noémie (2003). Descriptions d'ambiances réelles et virtuelles : Analyses linguistique et cognitive de différents dispositifs de représentation d'un habitacle automobile, Mémoire de DEA de Sciences du Langage, Université Paris 3.

Clark, Herbert \& Wilkes-Gibbs, Deanna (1986). « Referring as a collaborative process », Cognition 22, 1-39.

Conklin, Harold (1964). « Hanunoo color categories », Hymes, Dell (éd.) Language in culture and society: a reader in linguistic and anthropology, New York, Evanston London, Harper \& Row, 189-192.

David, Sophie, Dubois, David, Rouby, Catherine \& Schaal, Benoît (1997). « L'expression des odeurs en français : analyse lexicale et représentation cognitive », Intellectica 1(24), 51-83.

Dubois, Danièle (1989). «Psychologie du langage, psycholinguistique, psychologie cognitive. Contribution de la psychologie aux sciences du langage », Histoire, Épistémologie, Langage, numéro spécial Sciences du langage et recherches cognitives 11, 85-104.

Dubois, Danièle (2000). « Categories as Acts of Meaning: The Case of Categories in Olfaction and Audition », Cognitive Science Quarterly 1, 35-68.

Dubois, Danièle (2002) Informations, représentations, connaissances, et significations : des objets en question dans les sciences cognitives, Berthelin, Jean-Baptiste (éd.), Du sujet : théorie et praxis, Maison des Sciences de l'Homme, Paris, 97-106.

Dubois, Danièle (2007). "From psychophysics to semiophysics: Categories as acts of meaning. A case study from olfaction and audition, back to colors », Plümacher, Martina \& Holz, Peter (eds), Speaking of Colors and Odors, Amsterdam, John Benjamins, 167-184.

Dubois, Danièle (2008). "Sens communs et sens commun : Expériences sensibles, connaissance(s) ou doxa ? », Langue Française 170, numéro spécial, Georges-Elia (éd.), Discours et sens commun, 41-53.

Dubois, Danièle (éd.) (2009). Le sentir et le dire : concepts et méthodologies en linguistique et psychologie cognitive, Paris, L'Harmattan.

Dubois, Danièle \& Cance, Caroline (2009). "Mettre un terme aux couleurs de base: déconstruction d'un paradigme dominant », Dubois, Danièle (éd.), Le sentir et le dire : concepts et méthodologies en linguistique et psychologie cognitive, Paris, L'Harmattan, 75-104.

Dubois, Danièle \& Grinevald, Colette (2003). « En voir de toutes les couleurs : processus de dénomination des couleurs et constructions cognitives », Vandeloise, Claude (éd.), Traité des sciences cognitives, Paris, Hermes-Lavoisier, 80-114.

Dubois, Danièle \& Poitou, Jacques (2002). «Les "normes catégorielles" : catégories sémantiques et/ou listes de mots. Structuration des catégories sémantiques entre le cognitif et le linguistique », Intellectica $2: 217-249$.

Dubois, Danièle \& Resche-Rigon, Philippe (1993). «Prototypes ou stéréotypes : productivité et figement d'un concept », Plantin, Christian (éd.), Lieux communs, Topoï, stéréotypes, clichés, Paris, Kimé, 372-389.

Dubois, Danièle \& Resche-Rigon, Philippe (1995). « De la "naturalité" des catégories sémantiques. Des catégories d'“objets naturels" aux catégories lexicales », Intellectica 1 , 217-245.

Dubois, Danièle \& Rouby, Catherine (2002). « Names and Categories for Odors: the "veridical Label" », Rouby, Catherine, Schaal, Benoît, Holley, André, Dubois, Danièle \& Gervais Rémi (eds), Olfaction, Taste, and Cognition, New York, Cambridge University Press, 47-66. 
Dubois, Danièle, Rouby, Catherine \& Sicard, Gilles (1997). «Catégories sémantiques et sensorialités : de l'espace visuel à l'espace olfactif », Enfance 1, numéro spécial L'odorat chez l'enfant, 141-151.

Dubois-Charlier, Françoise \& Galmiche, Michel (eds) (1972). «La sémantique générative », Langages 27.

Eco, Umberto (1976, éd. fr. 1992). La production des signes, Paris, Le Livre de poche.

Eco, Umberto (1994). La recherche de la langue parfaite, Paris, Le Seuil.

Evans, Nicholas \& Levinson, Stephen C. (2009). " The myth of language universals: Language diversity and its importance for cognitive science ». Behavioral and Brain Sciences 32(05), 429-448.

Fauconnier, Gilles (1985). Mental Spaces, Cambridge, MIT Press.

Fodor, Jerry (1983). The Modularity of Mind: An Essay on Faculty Psychology, Cambridge, MIT Press.

Foley, William A. (1997). Anthopological linguistics, Malden, Blackwell.

Fortis, Jean-Michel (1996). «Sémantique cognitive et espace », Rastier, François (éd.), Sens et Textes, Paris, Didier, 167-197.

Fortis, Jean-Michel (2011). «Comment la linguistique est (re)devenue cognitive », Revue d'Histoire des Sciences Humaines 25, 105-126.

Geeraerts, Dick (1993). «Des deux côtés de la sémantique structurale: sémantique historique et sémantique cognitive », Histoire Épistémologie Langage 15(1), 111-129.

Giboreau Agnès, Egoroff Carine, Guerrand Sylvie, Urdapilleta Isabel, Candel Danielle, Dacremont Catherine \& Dubois, Danièle (2007). « Defining sensory descriptors: towards writing guidelines based on terminology », Food Quality and preferences 18(2), 265-274.

Gibson, James (1979). The ecological Approach to Visual Perception, Boston, Houghton Mifflin.

Glynn, Dylan \& Fischer, Kerstin (eds) (2010). Quantitative methods in cognitive semantics: Corpus-driven approaches, Berlin, De Gruyter.

Guastavino, Catherine, Katz, Brian, Dubois, Danièle \& Polack, Jean-Dominique (2005). «Ecological Validity of Soundscape Reproduction », Acta Acustica 91, 333-341.

Guastavino, Catherine, (2009). «Validité écologique des dispositifs expérimentaux », Dubois, Danièle (éd.), Le Sentir et le Dire. Concepts et méthodes en psychologie et linguistique cognitives, Paris, L'Harmattan, 229-248.

Guedou, Georges \& Coninckx, Claude (1986). « La dénomination des couleurs chez les Fon », Journal de la société des Africanistes 56, 64-85.

Guignard, Jean-Baptiste (2011). «Linguistique Cognitive et modèles catégoriaux : Quelques considérations épistémologiques », CORELA 9(2). [En ligne] http://corela.edel.univpoitiers.fr/index.php?id=2127.

Howes, David (éd.) (1991). The Varieties of Sensory experience: A source book in the Anthropology of the Senses, Toronto, Toronto University Press.

Hutchins, Edwin (1995). Cognition in the wild, Cambridge, MIT Press.

Indergand, Michel (1994). « De l'expérience matérielle à l'expérience immatérielle de la couleur », Junod, Philippe \& Pastoureau, Michel (eds), La couleur : regards croisés sur la couleur du Moyen Âge au XXe siècle, Paris, Le léopard d'or.

Kristol, André (1978). Color. Les langues romanes devant le phénomène de la couleur, Berne, Francke.

Lakoff, George (1970). «Linguistics and natural logic », Synthèse 22, 151-271.

Lakoff, George (1971). « On generative semantics », Steinberg, Danny D. \& Jakobovits, Leon A. (eds), Semantics. An interdisciplinary reader in Philosophy, Linguistics and Psychology. Cambridge, Cambridge University Press, 232-296.

Lakoff, George (1987). Women, fire, and dangerous things, Chicago, Chicago University Press.

Lakoff, George \& Johnson, Mark (1980). Metaphors we live by, Chicago, The University of Chicago Press. 
Lakoff, George \& Johnson, Mark (1999). Philosophy in the flesh: the embodied mind and its challenge to Western thought, New York, Basic Books.

Langacker, R Ronald W. (1986) « An Introduction to Cognitive Grammar », Cognitive Science X, 1, 1-40.

Langacker, Ronald W. (1987). Foundations of Cognitive Grammar, Stanford, Stanford University Press.

Latour, Bruno (1993). « Le topofil de Boa-Vista ou la référence scientifique : montage photo-philosophique », Raisons pratiques 4, 187-216.

Leroi-Gourhan, André (1964) Le Geste et la Parole, 1: Technique et langage, Paris, Albin Michel, 1964-1965.

Levinson, Stephen C. (2001). «Yéli Dnye and the Theory of Basic Color Terms», Journal of Linguistic Anthropology 10 (1), 3-55.

Lindsay, Peter \& Norman, Donald (1972). Human Information Processing. An introduction to psychology, New York, Academic Press.

Lucy, John A. (1992). Language diversity and Thought, Cambridge, Cambridge University Press.

Lucy, John A. (1997). «The linguistics of "color" », Hardin Clyde L. \& Maffi Luisa (eds), Color categories in thought and language, Cambridge, Cambridge University Press, 320-345.

Majid, Asifa \& Levinson, Stephen C. (2011). « The Senses in Language and Culture », The Senses and Society 6, 5-18.

MacLaury, Robert E. (1997). Color and Cognition in Mesoamerica: Constructing Categories as Vantages, Austin, University of Texas Press.

Merleau-Ponty, Maurice (1934). "La nature de la Perception », conférence devant la Société française de philosophie, 21 avril 1934, Merleau-Ponty, Maurice (1996), Le primat de la perception, Paris, Verdier.

Mondada, Lorenza \& Dubois, Danièle (1995). « Construction des objets de discours et catégorisation : une approche des processus de référenciation ", Tranel, Travaux Neuchâtelois de Linguistique 23, 273-302.

Neisser, Ulric (1987). Concepts and conceptual Development: Ecological and intellectual factors in categorization, Cambridge, Cambridge University Press.

Nyckees, Vincent (2007). «La cognition humaine saisie par le langage : De la sémantique cognitive au médiationnisme », CORELA, numéro thématique Cognition, discours, contextes. [En ligne] http://corela.edel.univ-poitiers.fr/index.php?id=1538.

Paveau, Marie-Anne (2007). « Discours et cognition : les prédiscours entre cadres internes et environnement extérieur », CORELA, numéro thématique Cognition, discours, contextes. [En ligne] http://corela.edel.univ-poitiers.fr/index.php?id=1550.

Rastier, François (éd.) (1989). « Sciences du langage et recherches cognitives », Histoire Épistémologie Langage 11(1), numéro spécial.

Rastier, François (1990): « La triade sémiotique, le trivium et la sémantique linguistique», Nouveaux Actes Sémiotiques 9, 5-40.

Rastier, François (1991). Sémantique et recherches cognitives, Paris, PUF.

Rastier, François (1993). «La sémantique cognitive - éléments d'histoire et d'épistémologie», Histoire Épistémologie Langage 15(1), Histoire de la sémantique : 1890-1990, 153-187.

Rastier, François (2001). Arts et sciences du texte, Paris, PUF.

Rastier, François (2005). « Sémiotique du cognitivisme et sémantique cognitive : Questions d'histoire et d'épistémologie », Texto ! [En ligne]. http://www.revue-texto.net/Inedits/ Rastier/Rastier_Semantique-cognitive.html.

Resche-Rigon, Philippe (2009). «Dire ou vouloir dire : du langage idéal au bricolage des langues », Dubois, Danièle (éd.), Le sentir et le dire : concepts et méthodes en psychologie et linguistique cognitives, Paris, L'Harmattan.

Rosch-Heider, Eleanor. R. (1971). « Focal color areas and the development of color names », Developmental Psychology 4, 447-455.

Rosch, Eleanor (1973). « Natural categories », Cognitive psychology 4(3), 328-350.

Rosch, Eleanor (1978). « Principles of categorization », Rosch, Eleanor \& Lloyd, Barbara B. (eds), Cognition and categorization, Hillsdale, NJ, Lawrence Erlbaum Associates, 27-48. 
Rosch, Eleanor, Mervis, Carolyn., Gray, Wayne, Johnson, David \& Boyes-Braem, Penny (1976). Basic Objects in Natural Categories, Cognitive Psychology 8, 182-439.

Sahlins, Marshall (1976). Colors and cultures, Semiotica 16, 1-22.

Saunders, Barbara (1992). The Invention of Basic Colour Terms, Utrecht, ISOR.

Selosse, Philippe (2008). «Traduire la nomenclature botanique néo-latine de la Renaissance : la linguistique au secours de l'histoire des sciences », Duris, Pascal (éd.), Traduire la science hier et aujourd'hui, Pessac, Publications de la Maison des sciences de l'homme d'Aquitaine, 25-43.

Straus, Erwin (1935). Vom Sinn der Sinne, Berlin, Springer Verlag. Édition française (2000). Du sens des sens, Grenoble, Éditions Jérôme Million.

Suchman, Lucy A. (1987). Plans and situated actions. The problem of human-machine communication, Cambridge, Cambridge University Press.

Sweetser, Eve (1990). From Etymology to Pragmatics: Metaphorical and Cultural Aspects of Semantic Structure, Cambridge/New York, Cambridge University Press.

Talmy, Leonard (1992). "Les relations entre grammaire et cognition », Cahiers de Praxématique 18, 13-74.

Teubert, Wolfgang (2010). « Corpus Linguistics: An Alternative », Semen [En ligne] http:// semen.revues.org/8912.

Tognini Bonelli, Elena (2001). Corpus Linguistics at Work, Amsterdam, Benjamins.

Tort, Patrick (1989). La raison classificatoire, Paris, Aubier.

Van Dijk, Teun (1993). " Discourse and cognition in society », Crowley David \& Mitchell David (eds), Communication Theory Today, Oxford, Pergamon Press, 107126.

Varela, Francisco, Thomson, Evan \& Rosch, Eleanor (1991). The Embodied Mind: Cognitive Science and Human Experience, Cambridge, MIT Press.

Vignaux, Georges (1999). Le démon du classement : penser, organiser, Paris, Seuil.

Vion, Robert (2009). « De la subjectivité en discours aux discours sur la subjectivité », Dubois, Danièle (éd.), Le sentir et le dire : concepts et méthodologies en linguistique et psychologie cognitive, L'Harmattan, Paris, 357-391.

Warnier, Jean-Pierre (1999). Construire la culture matérielle. L'homme qui pensait avec ses doigts, Paris, PUF.

Wierzbicka, Anna (1988). The Semantics of Grammar, Amsterdam/Philadelphia, Benjamins.

Wierzbicka, Anna (2008). « Why there are no "colour universals" in language and thought», Journal of the Royal Anthropological Institute 14, 403-421.

Wyler, Siegfried (2007). « Color terms between elegance and beauty. The verbalization of color with textiles and cosmetics », Plümacher, Martina \& Holz, Peter (eds), Speaking of Colors and Odors, Amsterdam, John Benjamins, 113-118. 